University of Wollongong

Research Online

Faculty of Engineering and Information

Faculty of Engineering and Information

Sciences - Papers: Part B

Sciences

2018

Spatial data compression via adaptive dispersion clustering

Yuliya Marchetti

California Institute of Technology

Hai Nguyen

California Institute of Technology

Amy Braverman

California Institute of Technology, amy.braverman@jpl.nasa.gov

Noel A. Cressie

University of Wollongong, California Institute of Technology, ncressie@uow.edu.au

Follow this and additional works at: https://ro.uow.edu.au/eispapers1

Part of the Engineering Commons, and the Science and Technology Studies Commons

Research Online is the open access institutional repository for the University of Wollongong. For further information contact the UOW Library: research-pubs@uow.edu.au 


\title{
Spatial data compression via adaptive dispersion clustering
}

\author{
Abstract \\ Spatial Dispersion Clustering (ASDC), a new method of spatial data compression, is specifically designed \\ to reduce the size of a spatial dataset in order to facilitate subsequent spatial prediction. Unlike traditional \\ data and image compression methods, the goal of ASDC is to create a new dataset that will be used as \\ input into spatial-prediction methods, such as traditional kriging or Fixed Rank Kriging, where using the \\ full dataset may be computationally infeasible. ASDC can be classified as a lossy compression method \\ and is based on spectral clustering. It aims to produce contiguous spatial clusters and to preserve the \\ spatial-correlation structure of the data so that the loss of predictive information is minimal. An extensive \\ simulation study demonstrates the predictive performance of these adaptively compressed datasets for \\ several scenarios. ASDC is compared to two other data-reduction schemes, one using local \\ neighborhoods and one using simple binning. An application to remotely sensed sea-surface-temperature \\ data is also presented, and computational costs are discussed. \\ Disciplines \\ Engineering | Science and Technology Studies \\ Publication Details \\ Marchetti, Y., Nguyen, H., Braverman, A. \& Cressie, N. (2018). Spatial data compression via adaptive \\ dispersion clustering. Computational Statistics and Data Analysis, 117 138-153.
}

This journal article is available at Research Online: https://ro.uow.edu.au/eispapers1/1992 


\title{
Spatial data compression via adaptive dispersion clustering
}

\author{
Yuliya Marchetti ${ }^{\mathrm{a}, *}$, Hai Nguyen ${ }^{\mathrm{a}}$, Amy Braverman ${ }^{\mathrm{a}}$, Noel Cressie , $^{\mathrm{b}, \mathrm{a}}$ \\ ${ }^{a}$ Jet Propulsion Laboratory, Pasadena, CA, USA \\ ${ }^{b}$ University of Wollongong, Wollongong, Australia
}

\begin{abstract}
In this article, we introduce a method of spatial data compression, which we call Adaptive Spatial Dispersion Clustering (ASDC). It is specifically designed to reduce the size of a spatial dataset in order to facilitate subsequent spatial prediction. Unlike with traditional data and image compression methods, the goal of ASDC is to create a new dataset that will be used as input into spatial prediction methods, such as traditional kriging or Fixed Rank Kriging, where using the full dataset may be computationally infeasible. ASDC can be classified as a lossy compression method and is based on spectral clustering. It aims to produce contiguous spatial clusters and to preserve the spatial correlation structure of the data so that the loss of predictive information is minimal. Through simulations, we demonstrate the predictive performance of these adaptively compressed datasets for several scenarios. ASDC is compared to two other data-reduction schemes, one using local neighborhoods and one using simple binning. We also present an application to remotely sensed sea-surface temperature data.
\end{abstract}

Keywords: spatial data compression, spectral clustering, spatial clusters, spatial dispersion function

\section{Introduction}

Very large spatial and spatio-temporal datasets are becoming more commonplace in social, commercial, and scientific research. In the social and commercial realms this is largely due to the expansion of the internet, and the computerization of many aspects of daily life. In science, new technologies for data collection and experimentation have led to the demand for new analysis methods specifically designed for new data types. One area where this is

\footnotetext{
${ }^{*}$ Corresponding author

Email addresses: yuliya.marchetti@jpl.nasa.gov (Yuliya Marchetti), hai.nguyen@jpl.nasa.gov (Hai Nguyen), amy.j.braverman@jpl.nasa.gov (Amy Braverman), ncressie@uow.edu.au (Noel Cressie)
} 
especially true is Earth Science, where satellite remote sensing data play an increasingly important role in understanding the physics of the Earth's system and interactions among its components. Remote sensing data can be massive, with hundreds of millions to billions of data points collected per day, but at the same time they can be sparse, with gaps in coverage due to orbit patterns and observing technology limitations.

Spatial and spatio-temporal statistical inference methods are key to obtaining maximum scientific return from these data, but massiveness poses a serious challenge to conventional spatial statistical modeling approaches. It is natural to look for ways to make the computations more efficient, and various methods based on simplification of the statistical model have been proposed. Some enforce sparsity on large spatial covariance matrices (Furrer et al., 2006; Kaufman et al., 2008) or precision matrices (Besag and Kooperberg, 1995; Rue and Held, 2005; Lindgren et al., 2011; Eidsvik et al., 2014; Datta et al., 2015; Gramacy and Apley, 2015; Nychka et al., 2015), and others use dimension reduction to reduce the number of parameters required to specify covariance (Banerjee et al., 2008; Cressie and Johannesson, 2008; Finley et al., 2009; Sang and Huang, 2012; Nguyen et al., 2012). However, with everincreasing data collection capabilities, the majority of these methods by themselves may not be enough because they still require holding large matrices, e.g., basis-function matrices, in memory. The method presented in this article, Adaptive Spatial Dispersion Clustering (ASDC), takes a different approach that is intended to complement dimension-reduction and sparse methods: make the data smaller in a way that preserves the essential information required for good spatial prediction.

The idea of making data smaller while preserving their essential characteristics is not new. When a spatial dataset is massive, such as is the case for high-resolution global remote sensing data, spatial prediction could be performed by limiting the data to a small region of interest, and ignoring the rest. In fact, local kriging (Haas, 1990; Cressie, 1993; Hammerling et al., 2012) and similar methods rely on such an approach. An alternative is to use compressed data instead, where compression here means that the data outside the region of interest have been aggregated to coarse resolution. This approach could be advantageous if aggregation is done in a way that preserves spatial information and produces globally valid spatial covariance structures. "Gridding" or "binning," in which the entire spatial field is aggregated to a coarse spatial resolution, is a form of naive data reduction that does not explicitly address the preservation of spatial covariance.

Clustering is a foundation of traditional data compression, but spatial dependence is usually not incorporated directly into the fidelity criterion. In the case of image compres- 
sion, there are approaches that do account for spatial dependencies and cluster coherence (Ambroise et al., 1997; Hu and Sung, 2006; Craddock et al., 2012), but the goal is to recreate an approximation that is visually indistinguishable from the original image, rather than to preserve spatial structure for purposes of inference per se. In contrast, ASDC explicitly incorporates key aspects of spatial covariance through the use of spatial dispersion functions (Sampson and Guttorp, 1992). For spatial predictions in a region of interest, data outside the region of interest are compressed by assigning the geographic locations associated with them to spatial clusters. Each spatial cluster is represented by the mean value of the data at its constituent locations. Cluster assignments are obtained by applying spectral clustering to a weighted similarity matrix that accounts for covariances among locations outside the region of interest, and covariances between locations inside and outside. This forces spatial contiguity, and causes clusters near the region of interest to be smaller than those far away because spatial covariance generally decreases with distance. In this paper, we demonstrate our method using simulated and real data of moderate size, rather then massive size, as a proof-of-concept.

The remainder of this paper is organized as follows. In Section 2 we provide an overview of and motivation for spatial data compression. We describe the ASDC algorithm and the set of concepts necessary to understand and explain its logic. In Section 3 we describe a simulation experiment that quantifies ASDC's performance versus local kriging and binning by comparing these three methods on synthetic data where the true spatial covariance function is known. Then, in Section 4, we apply the three methods to a relatively small sea-surface temperature dataset for which the true spatial covariance function is not known. This demonstrates the practical value of ASDC in real-world situations. Finally, Section 5 offers some conclusions, a discussion of computational challenges, and directions for future research.

\section{Methods}

Most methodologies applied to spatial data operate on the principle of the First Law of Geography (Tobler, 1970): locations that are closer to each other tend to behave more similarly than do those further apart. This implies that spatial covariance decays with geographic distance in a more or less continuous way. In other words, data from locations far away from a point or region of interest carry less information about the behavior of the process at the locations of interest than do data obtained at nearby sites. Consequently, compressing data from far away should have less affect on the precision and accuracy of 
optimal spatial predictors such as kriging predictors. In this section we formalize these ideas and describe how the ASDC algorithm combines them to provide a formal approach to spatial data compression.

\subsection{Framework}

Let $\left\{Y(\mathbf{v}): \mathbf{v} \in \mathcal{D} \subset \overline{\mathcal{D}} \subset \mathbb{R}^{2}\right\}$ be a hidden, real-valued process of interest on a twodimensional spatial domain, where $\mathcal{D}=\left\{\mathbf{v}_{1}, \ldots, \mathbf{v}_{N}\right\}, N$ is the size of $\mathcal{D}$, and $\overline{\mathcal{D}}$ is the union of basic areal units (BAUs). Following Nguyen et al. (2012), a BAU is a fine-scale spatial tile that represents the support of the smallest area for which inferences will be made. The entire spatial domain $\overline{\mathcal{D}}$ is tesselated into a set of these non-overlapping tiles: $\overline{\mathcal{D}}=\bigcup_{k=1}^{N} A_{k}$, and each BAU $A_{k}$ is uniquely identified with a spatial location, $\mathbf{v}_{k}$. Henceforth, when referring to a location $\mathbf{v}_{k}$, it is to be understood that we are referring to its corresponding BAU $A_{k}$ and vice versa.

Let $Z(\mathbf{s})$ be an observation at location $\mathbf{s}$, and assume that $Z(\mathbf{s})$ is the sum of the true spatial process, $Y(\mathbf{s})$, and measurement error, $\epsilon(\mathbf{s})$ :

$$
Z(\mathbf{s})=Y(\mathbf{s})+\epsilon(\mathbf{s}) ; \quad \mathbf{s} \in \mathcal{D},
$$

where $\epsilon(\cdot)$ is a mean-zero white-noise Gaussian process with variance, $\operatorname{var}(\epsilon(\cdot))=\sigma_{\epsilon}^{2}$. Suppose that data are observed at $\left\{\mathbf{s}_{1}, \ldots, \mathbf{s}_{n}\right\} \subset \mathcal{D}$, and define the vector of observations as $\mathbf{Z}=\left(Z\left(\mathbf{s}_{1}\right), \ldots, Z\left(\mathbf{s}_{n}\right)\right)^{T}$, where $n \leq N$. We are typically interested in inferring $Y\left(\mathbf{v}_{0}\right)$ at a new location $\mathbf{v}_{0} \in \mathcal{D}$ by minimizing the mean squared prediction error (MSPE):

$$
\operatorname{MSPE}\left(Y\left(\mathbf{v}_{0}\right), \hat{Y}\left(\mathbf{v}_{0}\right)\right)=E\left[\left(Y\left(\mathbf{v}_{0}\right)-\hat{Y}\left(\mathbf{v}_{0}\right)\right)^{2}\right]
$$

with respect to $\hat{Y}\left(\mathbf{v}_{0}\right)$. In the case of kriging, $\hat{Y}\left(\mathbf{v}_{0}\right)=\mathbf{a}^{T} \mathbf{Z}$ is a linear predictor of $Y\left(\mathbf{v}_{0}\right)$ based on all the available observations $\mathbf{Z}$, and we wish to minimize the MSPE with respect to $\mathbf{a} \in \mathbb{R}^{n}$. The optimal weights, a, are called kriging weights, which can be obtained by solving linear systems for each prediction location (e.g., Cressie, 1993). These are based on the covariance matrix $\mathbf{C}=\left(c_{i j}\right)_{n \times n}$, where $c_{i j}=\operatorname{cov}\left(Z\left(\mathbf{s}_{i}\right), Z\left(\mathbf{s}_{j}\right)\right)$, and on $\operatorname{cov}\left(Y\left(\mathbf{v}_{0}\right), Z\left(\mathbf{s}_{i}\right)\right)$. Since $n$ can be very, very large, it may not be feasible to compute a using all the data. Yet, it is still desirable to use as much of the dataset's information as possible, and rather than simply ignoring a portion of the elements of $\mathbf{Z}$, we will reduce its size with spatial data compression.

For the sake of realism, consider a block of prediction locations, $\mathcal{D}_{0}=\left\{\mathbf{v}_{0,1}, \ldots, \mathbf{v}_{0, M}\right\}$ 
of size $\left|\mathcal{D}_{0}\right|=M$. Then the rest of the locations form an exterior subdomain $\mathcal{D}_{e}=\mathcal{D} \backslash$ $\mathcal{D}_{0}$. The observed data vector $\mathbf{Z}$ can then be partitioned as $\mathbf{Z}=\left(\mathbf{Z}_{0}^{T}, \mathbf{Z}_{e}^{T}\right)^{T}$, where $\mathbf{Z}_{0}=$ $\left(Z\left(\mathbf{s}_{1}\right), \ldots, Z\left(\mathbf{s}_{m}\right)\right)^{T}$ and $\mathbf{Z}_{e}=\left(Z\left(\mathbf{s}_{m+1}\right), \ldots, Z\left(\mathbf{s}_{n}\right)\right)^{T}$, and where $\left\{\mathbf{s}_{1}, \ldots, \mathbf{s}_{m}\right\} \in \mathcal{D}_{0}$ and $\left\{\mathbf{s}_{m+1}, \ldots, \mathbf{s}_{n}\right\} \in \mathcal{D}_{e}$. We assume that the region of interest can always be chosen such that $\mathcal{D}_{0} \cap\left\{\mathbf{s}_{1}, \ldots, \mathbf{s}_{n}\right\} \neq \emptyset$, i.e. there is always at least one observed location.

Our goal is to perform spatial compression by clustering $N-M$ locations in $\mathcal{D}_{e}$ into $K$ disjoint subsets, $\Gamma_{e, j}$, with $\cup_{j=1}^{K} \Gamma_{e, j}=\mathcal{D}_{e}$ and where $K \ll n$ and is currently pre-specified by the user based on memory and other computational limitations. We then assign the $\ell$-th location in $\mathcal{D}_{0}$ to a $(K+\ell)$-th cluster, $\Gamma_{K+\ell}=\mathbf{v}_{0, \ell}$, such that $\cup_{\ell=1}^{M} \Gamma_{K+\ell} \cup \Gamma_{e}=\mathcal{D}$. We compute the aggregated data, $\boldsymbol{\Psi}=\left(\psi\left(\Gamma_{1}\right), \ldots, \psi\left(\Gamma_{K+M}\right)\right)^{T}$ from $\mathbf{Z}$; see (3) below. The subsequent statistical inference for $\mathcal{D}_{0}$ based on $\boldsymbol{\Psi}$ should be "similar" to that based on $\mathbf{Z}$.

A compression matrix is an $N \times(K+M)$ binary matrix $\mathbb{Q}$ in which the $k$-th row corresponds to the $k$-th location in $\mathcal{D}, k=1, \ldots, N$, and the $j$-th entry in that row corresponds to the $j$-th spatial cluster, $\Gamma_{j}, j=1, \ldots, K+M$. The $k, j$-th cell of $\mathbb{Q}$, denoted by $[\mathbb{Q}]_{k j}$, is 1 if the $k$-th location is assigned to $\Gamma_{j}$ and is 0 otherwise, with the proviso that $\sum_{j=1}^{K+M}[\mathbb{Q}]_{k j}=1$. The $j$-th spatial cluster is defined by

$$
\Gamma_{j}=\bigcup_{k=1}^{N}\left\{\mathbf{v}_{k}:[\mathbb{Q}]_{k j}=1\right\},
$$

and it is natural to define the spatial support of $\Gamma_{j}$ as the union of the spatial supports associated with its constituent locations: the support of $\Gamma_{j}$ is $\cup_{k=1}^{N}\left\{A_{k}:[\mathbb{Q}]_{k j}=1\right\}$.

Finally,

$$
\psi\left(\Gamma_{j}\right)=\frac{1}{n_{j}} \sum_{i=1}^{n} Z\left(\mathbf{s}_{i}\right) 1\left(\mathbf{s}_{i} \in \Gamma_{j}\right), \quad n_{j}>0,
$$

denotes the aggregated data for the $j$-th cluster; $j=1, \ldots, K+M$. In $(3), 1(\cdot)$ is the indicator function and $n_{j}=\sum_{i=1}^{n} 1\left(\mathbf{s}_{i} \in \Gamma_{j}\right)$. An aggregated value does not exist if $n_{j}=0$ (i.e. if there are no observations associated with the $j$-th cluster $\Gamma_{j}$ ).

Kriging using the available data $\mathbf{Z}$ in $\mathcal{D}$ would result in the full-kriging predictor, $\hat{Y}\left(\mathbf{v}_{0}\right)=$ $\mathbf{a}_{0}^{T} \mathbf{Z}$, for $\mathbf{v}_{0} \in \mathcal{D}_{0}$. Alternatively, the compressed kriging predictor is $\tilde{Y}\left(\mathbf{v}_{0}\right)=\mathbf{b}_{0}^{T} \mathbf{\Psi}$. Ideally, they give similar predictions. That is, ideally,

$$
\operatorname{MSPE}\left(\hat{Y}\left(\mathbf{v}_{0}\right), \tilde{Y}\left(\mathbf{v}_{0}\right)\right)=E\left[\left(\hat{Y}\left(\mathbf{v}_{0}\right)-\tilde{Y}\left(\mathbf{v}_{0}\right)\right)^{2}\right]=E\left[\left(\mathbf{a}_{0}^{T} \mathbf{Z}-\mathbf{b}_{0}^{T} \mathbf{\Psi}\right)^{2}\right] \approx 0,
$$


but the cost of computing $\mathbf{b}_{0}^{T} \boldsymbol{\Psi}$ is substantially less than that of $\mathbf{a}_{0}^{T} \mathbf{Z}$. Since $\hat{Y}\left(\mathbf{v}_{0}\right)$ is the best linear unbiased predictor, and $\tilde{Y}\left(\mathbf{v}_{0}\right)$ is linear in $\boldsymbol{\Psi}$ and hence in $\mathbf{Z}$, and it is unbiased, then $E\left[\left(\mathbf{a}_{0}^{T} \mathbf{Z}-\mathbf{b}_{0}^{T} \mathbf{\Psi}\right)^{2}\right] \geq 0$.

\subsection{Spatial dispersion function}

The central idea behind ASDC is to use the spatial dispersion function to perform spatial clustering in $\mathcal{D}_{e}$; that is, we specify $\mathbb{Q}$ in a way that the left-hand side of (4) is as small as possible. The spatial dispersion function is a smooth, non-negative function of geographic locations that indicates the spatial covariance structure of these locations (Sampson and Guttorp, 1992). Except for smoothness and non-negativity, we do not impose any other constraints on the spatial dispersion function and so, like Sampson and Guttorp (1992), we avoid using the term variogram. We exploit the spatial dispersion function to enforce fidelity to the underlying spatial covariance function, to induce spatial contiguity, and to adaptively determine the appropriate degree of compression throughout the spatial subdomain $\mathcal{D}_{e}$.

A measure of local spatial dispersion between any two locations $\mathbf{s}_{i}$ and $\mathbf{s}_{j}$ is the squared difference between the corresponding observed values $Z\left(\mathbf{s}_{i}\right)$ and $Z\left(\mathbf{s}_{j}\right)$ :

$$
d_{i j}^{2}=\left|Z\left(\mathbf{s}_{i}\right)-Z\left(\mathbf{s}_{j}\right)\right|^{2} .
$$

Define the spatial dispersion function $g_{\phi}(\cdot)$ as a smooth continuous function of distance between $\mathbf{s}_{i}$ and $\mathbf{s}_{j}, D\left(\mathbf{s}_{i}, \mathbf{s}_{j}\right)$, that depends on the spatial variability of $\mathbf{Z}$ expressed through parameters $\phi$. For example, $D\left(\mathbf{s}_{i}, \mathbf{s}_{j}\right)=\left\|\mathbf{s}_{i}-\mathbf{s}_{j}\right\|$, but other distances, such as great circle distance on the spheres are possible. The spatial dispersion function $g_{\phi}$ can assume any form as long as it is a smooth and non-negative function of distance. For example, it can be a specific parametric form used in spatial statistics or it can be a non-parametrically estimated function, such as a non-negative spline (Wever, 1988; Papp and Alizadeh, 2014). Like a variogram, the spatial dispersion function, $g_{\phi}$, can be fitted by minimizing

$$
\sum_{i} \sum_{j}\left|d_{i j}^{2}-g_{\phi}\left(D\left(\mathbf{s}_{i}, \mathbf{s}_{j}\right)\right)\right|^{2}
$$

with respect to $\phi$. This results in $\hat{\phi}$ and the fitted $g_{\hat{\phi}}$; see Sampson and Guttorp (1992) and Bornn et al. (2012), where spatial dispersion functions are used in optimal spatial estimation problems to construct covariance matrices for $\mathbf{Z}$. Here, we use the spatial dispersion function to inform spatial data compression and not directly for estimation or prediction.

Ultimately, we will determine the compression matrix $\mathbb{Q}$ by applying a spectral clustering 
algorithm to a collection of $N-M$ feature vectors, each representing one of the $N-M$ locations in $\mathcal{D}_{e}$. Section 2.4 explains how we derive the features and use them to populate a similarity matrix that is input into the clustering algorithm, but first we review the basics of spectral clustering in Section 2.3 below.

\subsection{Spectral clustering}

Spectral clustering (Shi and Malik, 2000; Ng et al., 2002) is a very popular method in unsupervised learning because of its simplicity and superior performance (Von Luxburg, 2007). Spectral clustering does not make any assumptions about the shape or structure of the resulting clusters, it captures non-spherical and non-convex clusters well, and it can also be applied to very large datasets (Chen et al., 2011; Song et al., 2008; Zare et al., 2010). See Von Luxburg (2007) for a summary of spectral clustering and a discussion of its relationship to spectral graph theory and graph partitioning problems.

We briefly outline the underlying methodology of spectral clustering. Let $\mathbf{x}_{1}, \ldots, \mathbf{x}_{n}$ be a set of data vectors, and for $i=1, \ldots, n$, let $w_{i j}$ represent a "similarity" measure between $\mathbf{x}_{i}$ and $\mathbf{x}_{j}$, where $w_{i j} \geq 0, w_{i j}=w_{j i}$. The goal of spectral clustering is to group the data vectors $\mathbf{x}_{1}, \ldots, \mathbf{x}_{n}$ into $K$ subsets using weights $w_{i j}$ such that data points within a subset are "similar" to each other, while data points in different subsets are "dissimilar". There are many options for calculating the similarity weights $w_{i j}$. For example, $w_{i j}$ can simply take values $\in\{0,1\}$, with $w_{i j}=0$ if the two data vectors $\mathbf{x}_{i}$ and $\mathbf{x}_{j}$ are not related and $w_{i j}=1$, if they are. The most commonly used weights are computed based on the Gaussian kernel:

$$
w_{i j}=\exp \left(-\left\|\mathbf{x}_{i}-\mathbf{x}_{j}\right\|^{2} / 2 \tau^{2}\right), \quad i, j=1, \ldots, n,
$$

where $\tau^{2}$ is called the scaling parameter and is usually defined by the user. The weights $w_{i j}$ are collected in a weighted similarity matrix $\mathbf{W}=\left[w_{i j}\right]_{n \times n}$, from which a normalized Laplacian matrix, $\mathbf{L}$, is computed:

$$
\mathbf{L}=\mathbf{I}-\mathbf{D}^{-1 / 2} \mathbf{W D}^{-1 / 2},
$$

where $\mathbf{D}$ is diagonal with the $i$ th diagonal element, $d_{i i}$, equal to the row sums of $\mathbf{W}$, and $\mathbf{I}$ is the identity matrix. That is,

$$
w_{i+}=\sum_{j=1}^{n} w_{i j} .
$$


It is apparent from Equation (8) that $\mathbf{L}$ is an $n \times n$ square matrix containing normalized dissimilarities.

The eigen-decomposition of $\mathbf{L}$ is given by,

$$
\mathbf{L}=\mathbf{Q}^{T} \Lambda \mathbf{Q}
$$

where $\mathbf{Q}$ is the $n \times n$ matrix with columns that are the eigenvectors of $\mathbf{L}$, and $\mathbf{\Lambda}$ is a diagonal matrix with the eigenvalues of $\mathbf{L}$ on the diagonal. Denote the columns of $\mathbf{Q}$ by $\mathbf{q}_{1}, \ldots, \mathbf{q}_{n}$ and the eigenvalues by $\lambda_{1}, \ldots, \lambda_{n}$. Assume that the eigenvalues are ordered from smallest to largest, $0=\lambda_{1} \leq \lambda_{2} \leq \lambda_{n}$ (Chung, 1997), and that columns of $\mathbf{Q}$ are in corresponding order so that $\mathbf{Q}=\left[\mathbf{q}_{1}, \ldots, \mathbf{q}_{n}\right]$. The truncated $n \times K$ matrix of eigenvectors is,

$$
\mathbf{U}=\left[\mathbf{q}_{1}, \ldots, \mathbf{q}_{K}\right]
$$

where $K$ is the number of clusters.

The final step of spectral clustering is to apply the $K$-means clustering algorithm (Hartigan and Wong, 1979; Arthur and Vassilvitskii, 2007) to the rows of U. Since the rows of U correspond to $\mathbf{x}_{1}, \ldots, \mathbf{x}_{n}$, the result is an assignment of each $\mathbf{x}_{i}$ to one of $K$ clusters on the basis of the similarities of $\mathbf{x}_{i}$ to one another in a space that emphasizes specific aspects of their relationships through $\mathbf{W}$. In the next section, we describe how we use this to cluster $N-M$ locations in $\mathcal{D}_{e}$ in a way that respects the spatial covariance characteristics in $\mathcal{D}_{e}$ for spatial compression as well as that between $\mathcal{D}_{e}$ and $\mathcal{D}_{0}$, as defined in Section 2.1.

\subsection{Adaptive spatial dispersion clustering}

To make good predictions at locations in $\mathcal{D}_{0}$ from locations in $\mathcal{D}_{e}$, it is necessary to preserve the covariance structure of the process between $\mathcal{D}_{0}$ and $\left\{\mathbf{v}_{e, i} \in \mathcal{D}_{e}: i=1, \ldots, N-M\right\}$. To adaptively compress locations in $\mathcal{D}_{e}$, it is also important to preserve the covariance structure of the process among all pairs of locations $\mathbf{v}_{e, i}$ and $\mathbf{v}_{e, j}, i, j=1, \ldots, N-M$. The weighted similarity matrix $\mathbf{W}$ discussed in Section 2.3 must incorporate both these covariance structures, and we achieve that by basing the weighted similarities on a combination of two different spatial dispersion functions that capture the two covariance structures.

To quantify spatial covariance structure among locations in $\mathcal{D}_{e}$ and between locations in $\mathcal{D}_{0}$ and in $\mathcal{D}_{e}$, we compute two sets of spatial dispersions as per $(5):\left\{d_{e, i j}^{2}=\left(Z\left(\mathbf{s}_{e, i}\right)-\right.\right.$ $\left.\left.Z\left(\mathbf{s}_{e, j}\right)\right)^{2}: \mathbf{s}_{e, i}, \mathbf{s}_{e, j} \in \mathcal{D}_{e}\right\}$ are spatial dispersions between locations in $\mathcal{D}_{e}$ and $\left\{d_{0, \ell k}^{2}=\right.$ $\left.\left(Z\left(\mathbf{s}_{0, \ell}\right)-Z\left(\mathbf{s}_{e, k}\right)\right)^{2}: \mathbf{s}_{0, \ell} \in \mathcal{D}_{0}, \mathbf{s}_{e, k} \in \mathcal{D}_{e}\right\}$ are spatial dispersions between $\mathcal{D}_{0}$ and locations in $\mathcal{D}_{e}$. Using (6) we estimate two spatial dispersion functions, $g_{\hat{\phi}_{e}}$ from $\left\{d_{e, i j}^{2}\right\}$ and $g_{\hat{\phi}_{0}}$ 
from $\left\{d_{0, \ell k}^{2}\right\}$. The appropriate parametric forms of $g_{\phi_{e}}$ and $g_{\phi_{0}}$ are problem-specific; in our applications, we have used both the exponential function and non-negative splines.

The weighted similarities for spectral clustering we use are defined by

$$
w_{i j}=\exp \left(-\frac{\left\|\mathbf{h}_{e, i}-\mathbf{h}_{e, j}\right\|^{2}}{2 \sqrt{h_{0, i}} \cdot \sqrt{h_{0, j}}}\right),
$$

where $\mathbf{h}_{e, i}$ and $\mathbf{h}_{e, j}$ are $(N-M)$-dimensional vectors derived from the spatial dispersion function $g_{\hat{\phi}_{e}}$ that models the spatial structure in $\mathcal{D}_{e}$, and $h_{0, i}$ and $h_{0, j}$ are scalars derived from the spatial dispersion function $g_{\hat{\phi}_{0}}$ that models the relationship between locations $\mathbf{v}_{e, i} \in \mathcal{D}_{e}$ and $\mathcal{D}_{0}$. Equation (12) is immediately recognizable as a form of the Gaussian kernel given by (7) with $\mathbf{x}_{i}$ and $\mathbf{x}_{j}$ replaced by $\mathbf{h}_{e, i}$ and $\mathbf{h}_{e, j}$, and $\tau^{2}$ replaced by $\left(\sqrt{h_{0, i}} \cdot \sqrt{h_{0, j}}\right)$. Next, we explain the derivations of $\mathbf{h}_{e, i}, \mathbf{h}_{e, j}, h_{0, i}$, and $h_{0, j}$.

Define the matrix $\mathbf{H}$ for which the $i j$-th element is

$$
[\mathbf{H}]_{i j}=g_{\hat{\phi}_{e}}\left(D\left(\mathbf{v}_{e, i}, \mathbf{v}_{e, j}\right)\right)
$$

for $i, j=1, \ldots, N-M$, where $D\left(\mathbf{v}_{e, i}, \mathbf{v}_{e, j}\right)$ is a distance measure between $\mathbf{v}_{e, i}$ and $\mathbf{v}_{e, j}$. The $i$-th row of $\mathbf{H}$ is a $(N-M)$-dimensional vector of spatial dispersions between location $\mathbf{v}_{e, i}$ and all other locations in $\mathcal{D}_{e}$. Thus, row $i$ of of $\mathbf{H}$ can be thought of as a basis expansion that encodes the available information about how the process at $\mathbf{v}_{e, i}$ covaries with all other locations in $\mathcal{D}_{e}$. In (12), $\mathbf{h}_{e, i}$ and $\mathbf{h}_{e, j}$ are obtained from the $i$-th and $j$-th rows of $\mathbf{H}$, respectively.

Define the geographic distance between location $\mathbf{v}_{e, i}$ and $\mathcal{D}_{0}$ to be the distance between $\mathbf{v}_{e, i}$ and the location in $\mathcal{D}_{0}$ that is nearest to $\mathbf{v}_{e, i}$ :

$$
D\left(\mathcal{D}_{0}, \mathbf{v}_{e, i}\right) \equiv D\left(\mathbf{v}_{e, i}^{*}, \mathbf{v}_{e, i}\right), \quad \text { where } \quad \mathbf{v}_{e, i}^{*}=\underset{\mathbf{v} \in \mathcal{D}_{0}}{\operatorname{argmin}} D\left(\mathbf{v}, \mathbf{v}_{e, i}\right) .
$$

Finally, the scaling parameter in the denominator of (12) is

$$
h_{0, i}=g_{\hat{\phi}_{0}}\left(D\left(\mathbf{v}_{e, i}^{*}, \mathbf{v}_{e, i}\right)\right) \text {, }
$$

which down-weights the exponent in (12) if the processes at locations $\mathbf{v}_{e, i}$ and $\mathbf{v}_{e, j}$ have large spatial dispersions relative to the prediction region $\mathcal{D}_{0}$. By using an adaptive scaling parameter $\tau^{2}=\left(\sqrt{h_{0, i}} \sqrt{h_{0, j}}\right)$, locations in $\mathcal{D}_{e}$ that are typically close to $\mathcal{D}_{0}$ are grouped into small clusters, while locations typically further from $\mathcal{D}_{0}$ will be compressed into large 
clusters. See Zelnik-Manor and Perona (2004) for a general discussion of adaptive scaling parameters.

Once the similarity matrix $\mathbf{W}$ is constructed from (12), we obtain the $(N-M) \times K$ matrix $\mathbf{U}$ as shown in (11) and apply the $K$-means algorithm to cluster its rows. The $K$-means algorithm partitions each row of $\mathbf{U}$ into $K$ clusters. The result is the binary matrix $\mathbb{Q}$ given in Section 2.1 and used for aggregation in (2) for Adaptive Spatial Dispersion Clustering (ASDC).

Figure 1 is an example of ASDC applied to a synthetic spatial field. Figure 1a is a simulated random field of size $N=14,400$ generated from a model specified by (1) with $\mathbf{Y} \sim N\left(\mathbf{0}, \sigma_{Y}^{2} \boldsymbol{\Sigma}\right)$ and $\boldsymbol{\epsilon} \sim N\left(\mathbf{0}, \sigma_{\epsilon}^{2} \mathbf{I}\right)$. The covariance matrix $\boldsymbol{\Sigma}$ is constructed from an exponential model,

$$
\sigma_{i j}= \begin{cases}\sigma_{Y}^{2} \exp \left\{-\frac{\left\|\mathbf{v}_{i}-\mathbf{v}_{j}\right\|}{\theta}\right\} & \text { if }\left\|\mathbf{v}_{i}-\mathbf{v}_{j}\right\|>0 \\ \sigma_{Y}^{2}+\sigma_{\epsilon}^{2} & \text { if }\left\|\mathbf{v}_{i}-\mathbf{v}_{j}\right\|=0\end{cases}
$$

with the spatial scale parameter $\theta=0.2, \sigma_{Y}^{2}=1$, and measurement error variance $\sigma_{\epsilon}^{2}=0.01$. Figure 1a shows simulated observations, $Z\left(\mathbf{s}_{i}\right)$ at each location $\mathbf{s}_{i} \in \mathcal{D}$. The small white square in the center identifies a prediction region $\mathcal{D}_{0}$ consisting of a single location $\mathbf{v}_{0}$. Figure $1 \mathrm{~b}$ shows the corresponding compressed data for $K=400$. The colors in Figure $1 \mathrm{~b}$ are the mean data values of clusters for all locations assigned to the same cluster, as encoded by matrix $\mathbb{Q}$. Very small or singleton clusters are formed around $\mathbf{v}_{0}$, and larger clusters

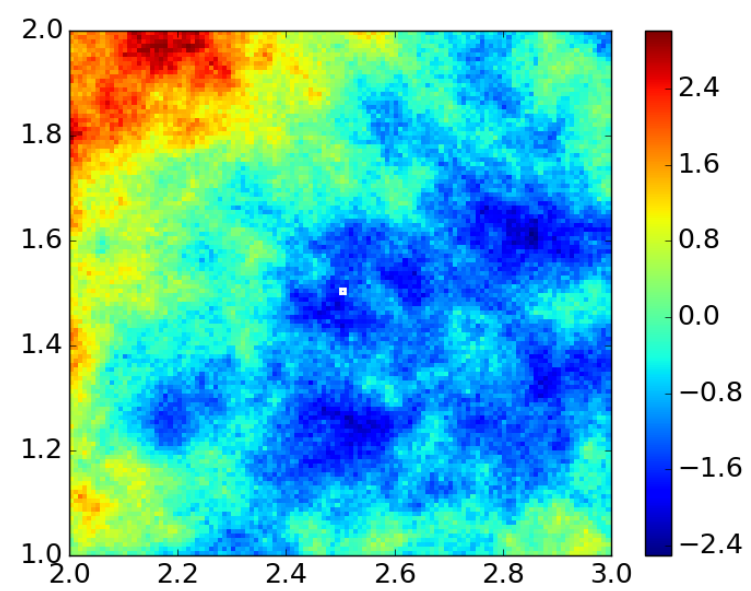

(a) simulated data

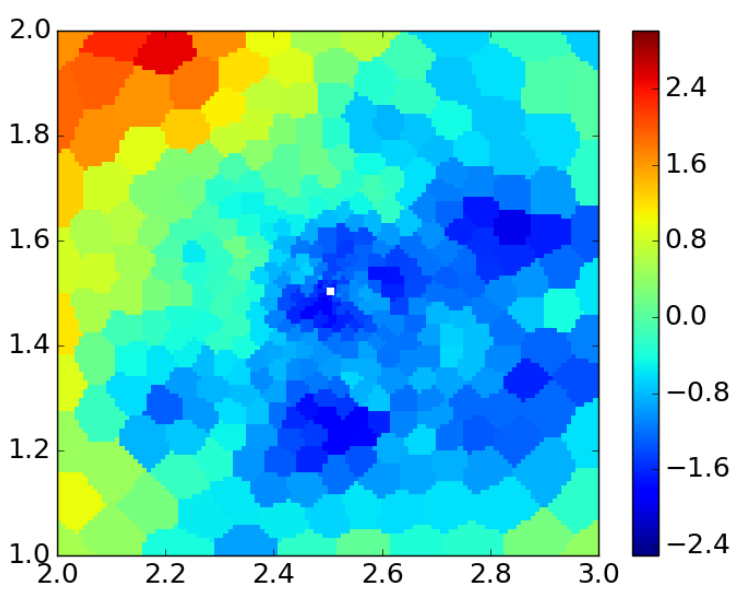

(b) compressed data

Figure 1: Simulated raw data (a) and the corresponding compressed data for $K=400$ (b). 


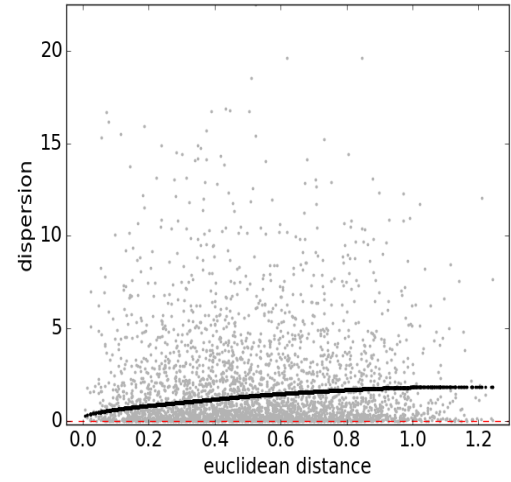

(a)

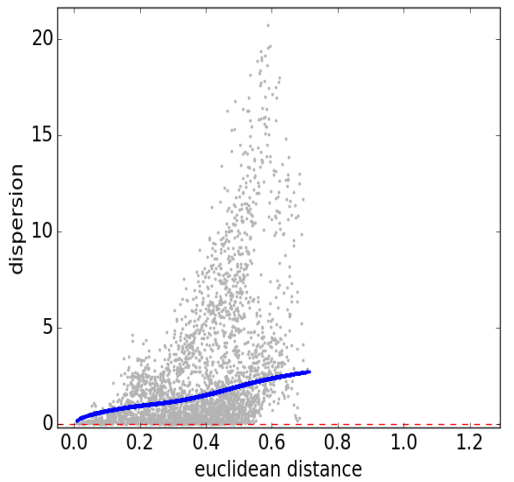

(b)

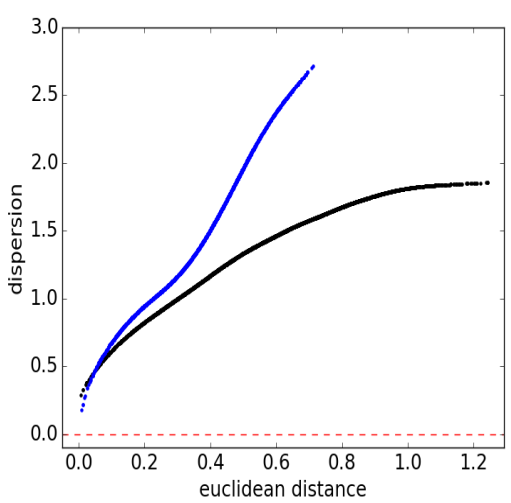

(c)

Figure 2: Spatial dispersions and non-parametrically fitted spatial dispersion functions. (a) spatial dispersions $\left\{d_{e, i j}^{2}\right\}$ (gray) and $g_{\hat{\phi}_{e}}$ (black), (b) spatial dispersions $\left\{d_{0, k}^{2}\right\}$ (gray) and $g_{\hat{\phi}_{0}}$ (blue), (c) spline dispersion functions $g_{\hat{\phi}_{e}}$ (black) and $g_{\hat{\phi}_{0}}$ (blue), where the vertical scale has been reduced.

are formed nearer to the edges of the domain. Smaller clusters preserve fine-scale spatial information around the prediction location.

Figures $2 \mathrm{a}$ and $2 \mathrm{~b}$ show the spatial dispersions and the corresponding estimated spatial dispersion functions for this simulated dataset. We used cubic splines for both $g_{\phi_{e}}$ and $g_{\phi_{0}}$ in order to demonstrate that spatial compression can be performed even without the knowledge of the exact covariance structure of the data. Figure 2c displays the two spatial dispersion functions $g_{\hat{\phi}_{e}}$ and $g_{\hat{\phi}_{0}}$ together for comparison and shows that the magnitude of the spatial dispersions for locations in $\mathcal{D}_{e}$ is smaller than that between the prediction location and locations in $\mathcal{D}_{e}$. We conclude that the spatial dependence between points in $\mathcal{D}_{e}$ is stronger than is the spatial dependence between $\mathbf{v}_{0}$ and locations in $\mathcal{D}_{e}$. This suggests favorable conditions for spatial data compression. If process at $\mathbf{v}_{0}$ was independent of the process in $\mathcal{D}_{e}$, then $\mathcal{D}_{e}$ would be compressible into a single cluster with no loss of information about the process at $\mathbf{v}_{0}$.

\subsection{Details of the ASDC algorithm}

We now summarize the algorithm for Adaptive Spatial Dispersion Clustering (ASDC): 


\section{Algorithm: ASDC}

Input: a block of prediction locations $\mathbf{v}_{0,1}, \ldots, \mathbf{v}_{0, M}$, a set of spatial locations $\left\{\mathbf{v}_{e}\right\}$, a set of observed spatial locations $\left\{\mathbf{s}_{1}, \ldots, \mathbf{s}_{n}\right\}$ and the corresponding observed data $\mathbf{Z}\left(\mathbf{s}_{1}\right), \ldots, \mathbf{Z}\left(\mathbf{s}_{n}\right)$, number of clusters $K$

1: Compute two sets of spatial dispersions $\left\{d_{e, i j}^{2}\right\}$ and $\left\{d_{0, \ell k}^{2}\right\}$ and the corresponding distances $D\left(\mathbf{s}_{e, i}, \mathbf{s}_{e, j}\right)$ and $D\left(\mathbf{s}_{0, \ell}, \mathbf{s}_{e, k}\right)$ :

a) $d_{e, i j}^{2}=\left(Z\left(\mathbf{s}_{e, i}\right)-Z\left(\mathbf{s}_{e, j}\right)\right)^{2}$, for $i, j=1, \ldots, n-m$

b) $d_{0, \ell k}^{2}=\left(Z\left(\mathbf{s}_{0, \ell}\right)-Z\left(\mathbf{s}_{e, k}\right)\right)^{2}$, for $k=1, \ldots, n-m, \ell=1, \ldots, m$

2: Fit $g_{\phi_{e}}$ and $g_{\phi_{0}}$ to $\left\{d_{e, i j}^{2}\right\}$ and $\left\{d_{0, \ell k}^{2}\right\}$, respectively, as per (6). That is, obtain estimates $\hat{\phi}_{e}$ and $\hat{\phi}_{0}$, resulting in:

a) $\left\{g_{\hat{\phi}_{e}}\left(D\left(\mathbf{v}_{e, i}, \mathbf{v}_{e, j}\right)\right): i, j=1, \ldots, N\right\}$

b) $\left\{g_{\hat{\phi}_{0}}\left(D\left(\mathbf{v}_{0, \ell}, \mathbf{v}_{e, k}\right)\right): \ell=1, \ldots, M, k=1, \ldots, N\right\}$

3: Construct $\mathbf{H}$ from (13) and $h_{0, i}$ from (14), for $i=1, \ldots N$

4: Construct $\mathbf{W}$ from (7)

5: Apply the spectral clustering algorithm to $\mathbf{W}$, and obtain cluster assignments

6: Construct clusters $\Gamma_{j}$ as in (2)

7: Compute cluster averages $\psi\left(\Gamma_{j}\right)$ per (3) to obtain the compressed dataset:

$$
\mathbf{\Psi}=\left(\psi\left(\Gamma_{1}\right), \ldots, \psi\left(\Gamma_{K+M}\right)\right)^{T}
$$

We demonstrate the performance of ASDC for inference and prediction in the next section. For the simulation, we make specific choices for $g_{\phi_{e}}$ and $g_{\phi_{0}}$ and use a block of prediction locations $\mathcal{D}_{0}$.

\section{Simulation experiments}

In this section we evaluate the performance of ASDC and compare it to that of two oftenused alternatives: subsetting and coarse-scale aggregation. Performance of a data reduction method is characterized by simulating an ensemble of synthetic spatial fields from a known exponential covariance function, withholding the data from $\mathcal{D}_{0}$ at the center of the spatial field to be used as a validation set, and applying kriging to the remaining data in $\mathcal{D}_{e}$, after compression, to predict the values in $\mathcal{D}_{0}$. The exponential function with known parameters is used to compute kriging covariance matrices as well as the parametric form of both $g_{\phi_{e}}$ 
and $g_{\phi_{0}}$. The quality of these predictions over $\mathcal{D}_{0}$ is quantified by the root mean squared prediction error,

$$
\mathrm{RMSPE}=\sqrt{\frac{1}{M} \sum_{i=1}^{M}\left(y\left(\mathbf{v}_{0, i}\right)-\hat{y}\left(\mathbf{v}_{0, i}\right)\right)^{2}}
$$

where $y\left(\mathbf{v}_{0, i}\right)$ is the true value of the process (with no measurement error) at prediction location $\mathbf{v}_{0, i}, i=1, \ldots, M, M=\left|\mathcal{D}_{0}\right|$, and $\hat{y}\left(\mathbf{v}_{0, i}\right)$ is the corresponding predicted value. We run the experiment four times using 1) the uncompressed (full) data in $\mathcal{D}_{e}, 2$ ) data in $\mathcal{D}_{e}$ reduced using ASDC, 3) data in $\mathcal{D}_{e}$ reduced by using only a subset of locations nearest to $\mathcal{D}_{0}$, and 4) data reduced by binning the data on a coarse spatial grid and then averaging by grid cell. For easy reference later, we refer to these four cases as "K-full", "K-adaptive", "Klocal", and "K-binned", respectively. In cases 2), 3), and 4), we ensure that the size of the compressed datasets is the same across cases so that differences in RMSPE reflect differences in the choice of compression strategy. The quality of each of the three compression methods is measured by their RMSPE's relative to that obtained in case 1), i.e., where there is no compression.

We perform a set of experiments to obtain distributions of RMSPE values for the three compression strategies, relative to the no-compression case, for a) several different spatial covariance structures in the underlying field, b) several different magnitudes of measurement errors in the observations, and c) two choices for the level of compression achieved. For each combination of a) and b), we generated 100 synthetic random spatial fields. Then, we compressed the data in $\mathcal{D}_{e}$ six times: using each of the three compression methods for both coarse and fine compression. Kriging was applied to $\mathcal{D}_{e}$ for the 100 simulations in each of the six ensembles, and to the uncompressed data in $\mathcal{D}_{e}$, which provides a benchmark. This yielded 100 RMSPE's for each combination of compression method and compression level, within each combination of spatial covariance structure and measurement error. Details of these steps are given below.

The experimental design crosses the four data-reduction strategies (including the fulldata case) with four spatial scale parameters for the underlying spatial model and five signal-to-noise values for the synthetic data. We summarize the results of these experiments graphically and with an analysis of variance that provides estimates of the main effects due to data reduction method, spatial scale, and signal-to-noise, and their two-way interactions. To assess robustness to the level of compression desired (parameterized by the choice of $K$ ), the analysis is performed for both fine $(K=600)$ and coarse $(K=200)$ compression. 


\subsection{Generating synthetic fields}

To create synthetic spatial fields, we generated stationary noisy data, according to (1), with $\mathbf{Y} \sim N\left(\mathbf{0}, \sigma_{Y}^{2} \boldsymbol{\Sigma}\right)$ for $\sigma_{Y}^{2}=1$ and $\boldsymbol{\epsilon} \sim N\left(\mathbf{0}, \sigma_{\epsilon}^{2} \mathbf{I}\right)$. The covariance matrix, $\boldsymbol{\Sigma}$, obtained from the isotropic exponential model in (15), where recall that $\theta$ is the spatial scale parameter. We generated fields of size $N=100 \times 100=10,000$ on $[0,1] \times[0,1]$ for all combinations of four spatial scale parameters $\theta=\{0.1,0.5,4,20\}$ and five measurement-error variances $\sigma_{\epsilon}^{2}=\{0.01,0.1,0.5,1.5,10\}$. Signal-to-noise ratio is defined by $\mathrm{SNR}=\sigma_{Y}^{2} / \sigma_{\epsilon}^{2}$. Thus, the measurement-error variances correspond to $\mathrm{SNR}=\{100,10,2,0.7,0.1\}$. For the mechanics of spatial field generation given a covariance structure, see for example Cressie (1993).

Examples of simulated complete fields for spatial scale parameters $\theta=\{0.5,4,20\}$ are shown in the top row of Figure 3. We omit $\theta=0.1$ in the figures since the result for this scale parameter were similar to those with $\theta=0.5$. The corresponding data with added measurement error and missing locations are shown in the second row of Figure 3. Since we anticipate that one important application of ASDC will be to remote sensing data, we chose $40 \%$ of the locations in each realization of the spatial field to be designated as missing and removed their data values. About half of the missing locations were chosen randomly and the other half were chosen around randomly generated "centers", and hence spherical missing areas are seen in the second row of Figure 3. The square outline in the center of the region shows the subdomain of interest, $\mathcal{D}_{0}$, where predictions are made. There are $M=$ $1089(33 \times 33)$ locations in $\mathcal{D}_{0}$. The simulated data for these locations are set aside and used later to evaluate the quality of the kriged predictions via RMSPE.

\subsection{Adaptive compression of synthetic fields}

The bottom two rows of Figure 3 show the ASDC-compressed data. The second row from the bottom shows coarse compression done with $K=200$ spatial clusters, and the bottom row shows fine compression with $K=600$ spatial clusters. This simulation used an SNR of 10. The locations in $\mathcal{D}_{e}$ with missing data are assigned to spatial clusters with the nearest geographic center, but they obviously do not contribute to the calculation of the cluster average.

Two features of the ASDC-compressed field are worth noting. First, spatial clusters near the subdomain of interest are typically smaller than those further away. This reflects the fact that spatial structure is maintained with greater fidelity at nearby locations than at locations further away, and the larger the spatial scale parameter, $\theta$, the larger is the set of "nearby" locations. Second, clusters tend to be more or less of the same geographic size for a given radial distance away from the center of $\mathcal{D}_{0}$. This feature is a consequence of the 


$$
\theta=0.5
$$
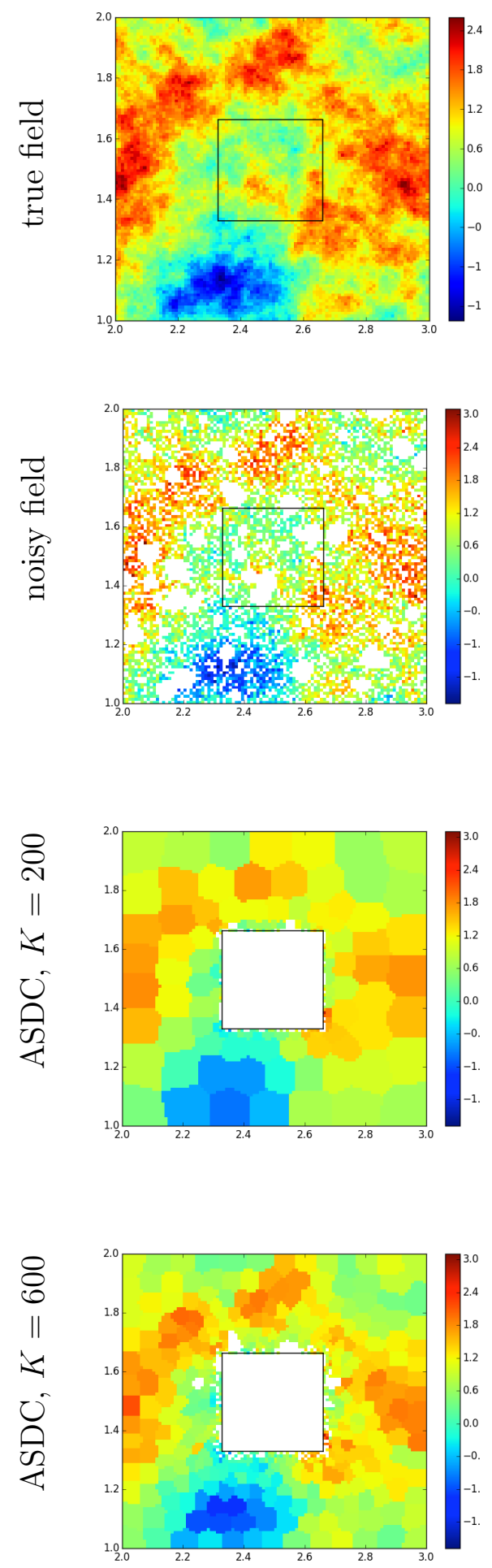

$\theta=4.0$
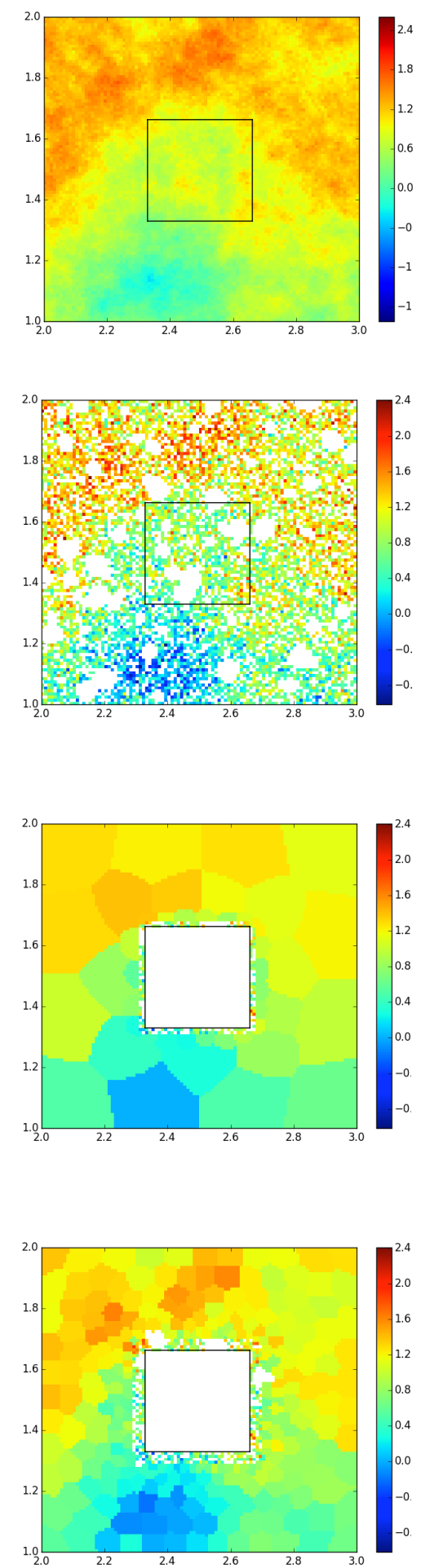

$\theta=20.0$
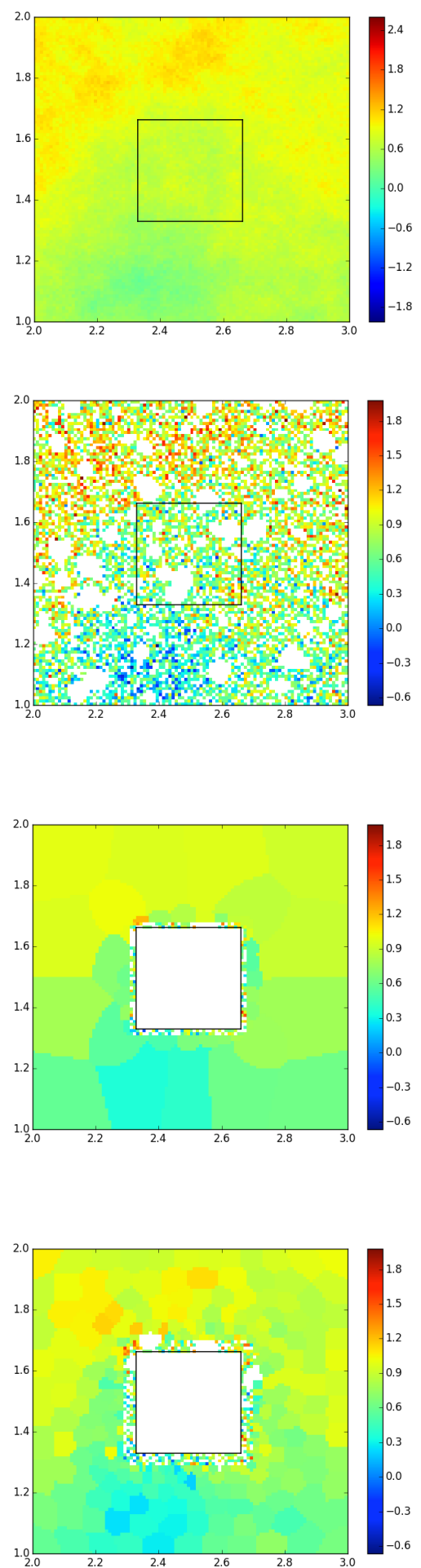

Figure 3: Top row : simulated true fields, $\theta=0.5$ (left), $\theta=4$ (center), and $\theta=20$ (right). Second row: corresponding noisy field with $\mathrm{SNR}=10$ and missing locations. Bottom two rows: corresponding ASDC-compressed fields for $K=200$ and $K=600$, where the region of interest inside the black square is $\mathcal{D}_{0}$. 
isotropic exponential function that is used to define the spatial clusters. The compressed data vector $\boldsymbol{\Psi}=\left(\psi\left(\Gamma_{1}\right), \ldots, \psi\left(\Gamma_{K}\right)\right)^{T}$ replaces the original data in $\mathcal{D}_{e}$, resulting in data-size reductions of $89 \%$ and $96 \%$, for the $K=600$ and $K=200$ cases, respectively, relative to the original size of $\mathcal{D}_{e}$.

Note also that for K-adaptive (i.e., ASDC), data values associated with locations in the compressed domain $\mathcal{D}_{e}$ can no longer be treated as point-referenced data, since they now have block support. This is also true for compressed data produced by K-binned. When kriging is applied to compressed fields produced by these two methods, the covariance matrices used in kriging must account for this block support. The spatial covariance matrices used to krige in the K-adaptive and K-binned cases can easily be computed using the bilinearity property of the covariance function:

$$
\bar{\sigma}_{k \ell}=\frac{1}{\left|C_{k}\right|\left|C_{\ell}\right|} \sum_{i^{\prime} \in C_{k}} \sum_{j^{\prime} \in C_{\ell}} \sigma_{i^{\prime} j^{\prime}}
$$

where $C_{k}$ and $C_{\ell}$ represent $k$-th and $\ell$-th clusters and $\sigma_{i^{\prime} j^{\prime}}$ is defined in (15).

\subsection{Experimental results}

Figure 4 shows the results of our simulation experiments. Each subplot represents the average RMSPE's, over the 100 trials of our experiments, with their $95 \%$ confidence intervals as functions of $\log _{10}(\mathrm{SNR})$. Figure $4 \mathrm{a}$ and $4 \mathrm{~b}$ show the results for fine $(K=200)$ and coarse ( $K=600)$ compression, respectively. The four panels in each row represent increasing spatial scale parameters in the underlying exponential model.

Although K-adaptive does not always achieve the lowest RMSPE, it is either the best, or nearly so, over the wide range of parameter choices used here. For a large SNR and across the four spatial scale parameters, K-adaptive and K-local show similar performance nearly comparable to that of K-full, and K-binned performs worse than the others, probably due to the fact that any possible strong correlations on the edges of $\mathcal{D}_{0}$ might be dampened by binning. As the spatial scale parameter increases (subplots from left to right) and the SNR decreases (along the $x$-axis), the performance of K-local deteriorates, especially for $K=200$, while the performance of K-binned improves. This might be because the longer correlation lengths mean that additional information from locations further away from $D_{0}$ is not being used by K-local, while it is being used by K-adaptive and K-binned to improve the predictions in the high-noise scenarios.

K-adaptive has the most stable performance across all scenarios. Even though K-adaptive performs only as well as K-local in low noise, short-scale scenarios, K-adaptive outperforms 

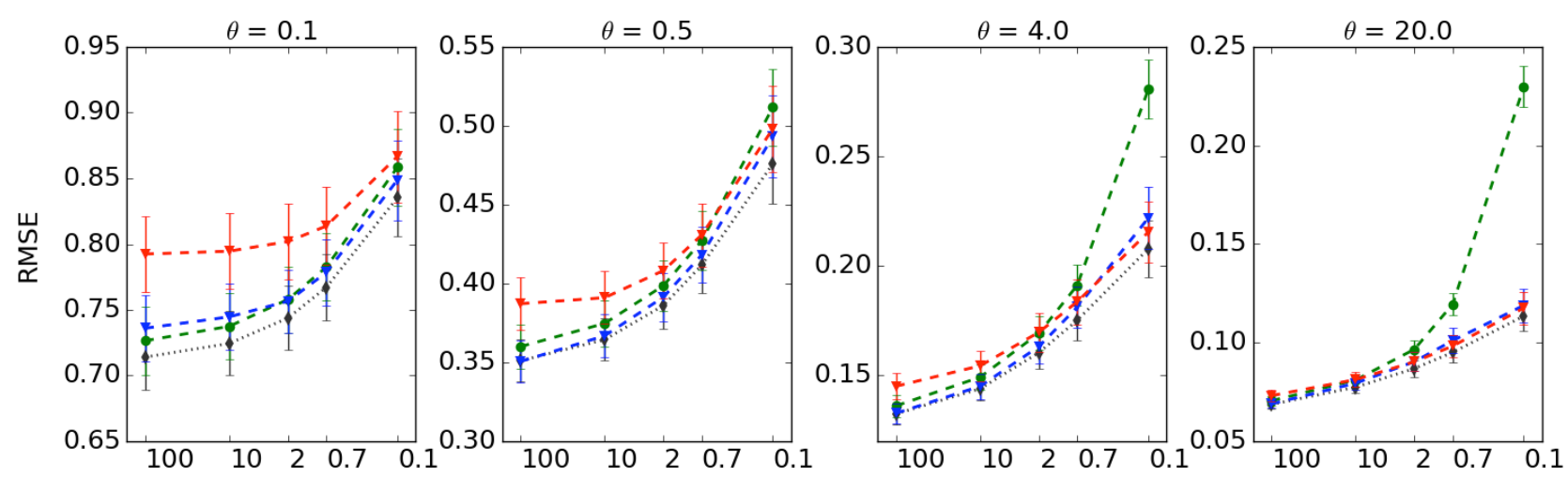

(a) $K=200$
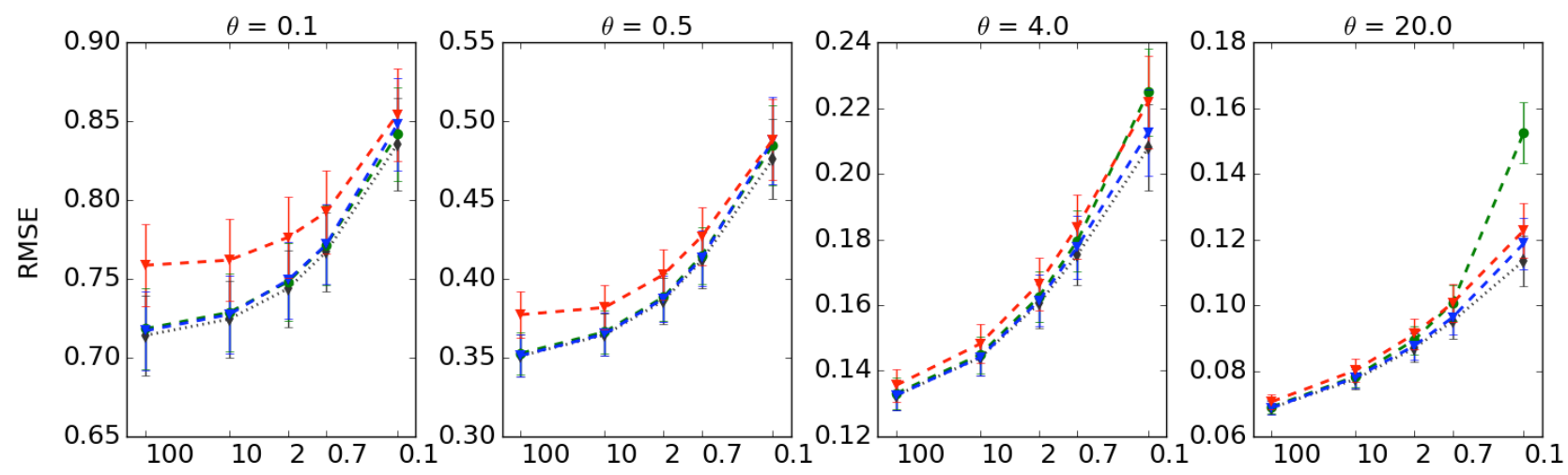

(b) $K=600$

Figure 4: Plot of the mean RMSPE and its 95\% confidence interval for K-adaptive (blue), K-local (green), K-binned (red), and K-full (black dotted line). Each subplot represents scale parameters $\theta=\{0.1,0.5,4,20\}$, respectively. On the $x$-axis is the logarithm of the $\mathrm{SNR}$, labeled as the actual SNR $=\{100,10,2,0.7,0.1\}$. On the $y$-axis is the mean RMSPE over 100 samples with its $95 \%$ confidence interval indicated by the error bars around the mean value.

K-local when data are noisy. Conversely, when SNR is low, K-adaptive and K-binned are comparable, but when SNR is high, K-binned's performance deteriorates, but K-adaptive's does not. In real-world applications, where we do not know the true values of $\theta$ or SNR, K-adaptive would be the choice with the lowest risk. Finally, K-adaptive achieves good performance and is relatively stable for both choices of $K$, and hence it may be especially useful in situations when high levels of data reduction are needed.

The preceding analysis focuses on the performance of the three candidate data reduction methods with respect to their predictions. It is equally important to ask how well these methods do in providing accurate estimates of prediction variance, which we measure via 


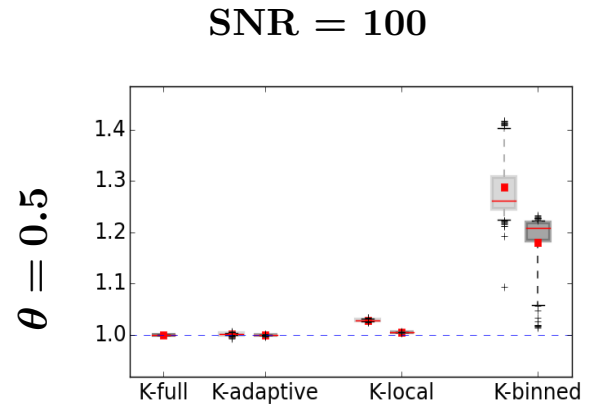

(a)

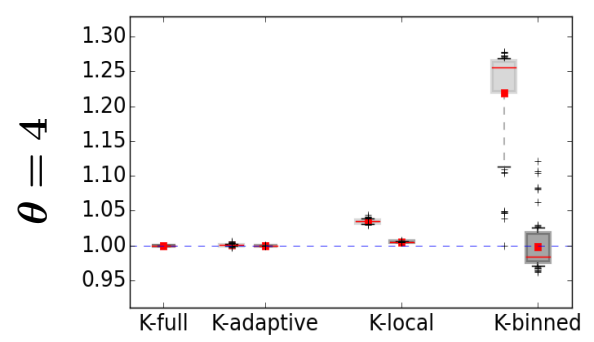

(d)

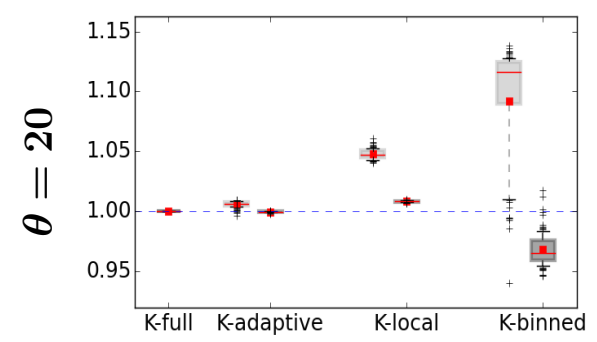

(g)
$\mathrm{SNR}=2$

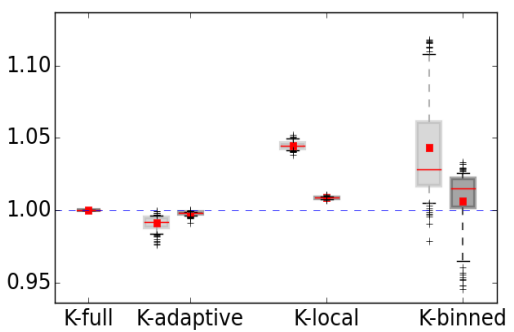

(b)

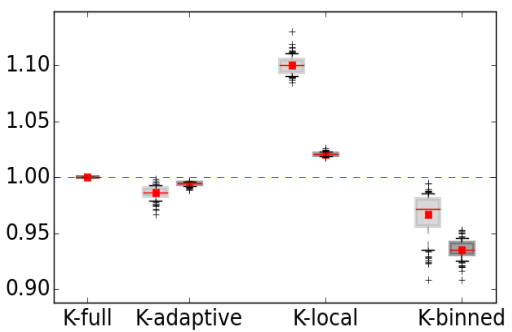

(e)

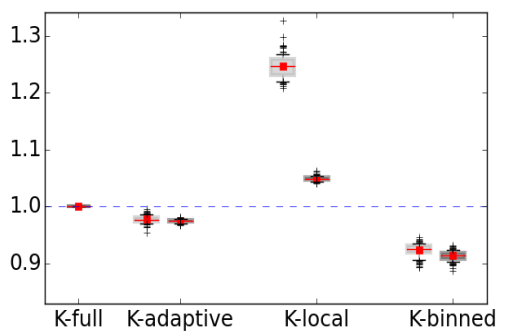

(h)
$\mathrm{SNR}=0.1$

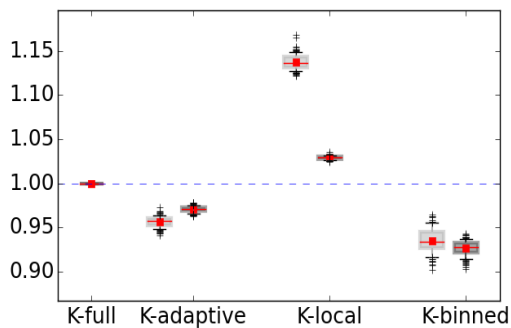

(c)

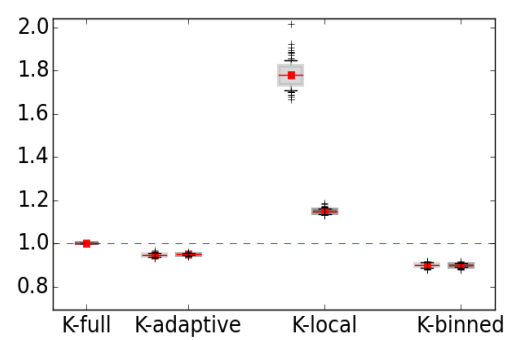

(f)

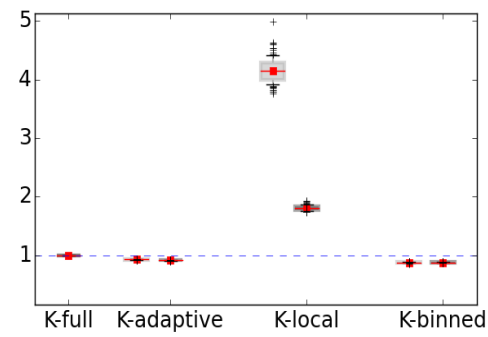

(i)

Figure 5: Boxplots of MPEVR for K-full, K-adaptive, K-binned, and K-local. Each row represents a different scale parameter $\theta$ and each column represents a different SNR. The color of the boxplots is as follows: dark gray color is for $\mathrm{K}$-full, light gray for $K=200$, and for medium gray for $K=600$. Values of the prediction error ratio closer to 1 are better.

mean prediction error variance ratio:

$$
\operatorname{MPEVR}=\frac{1}{M} \sum_{i=1}^{M} \frac{\operatorname{var}\left(\tilde{y}\left(\mathbf{v}_{0, i}\right)\right)}{\operatorname{var}\left(\hat{y}\left(\mathbf{v}_{0, i}\right)\right)}
$$

where $\tilde{y}\left(\mathbf{v}_{0, i}\right)$ represents a predicted value from $\mathrm{K}$-full, and $\hat{y}\left(\mathbf{v}_{0, i}\right)$ is a predicted value from kriging based on a compressed dataset. The boxplots in Figure 5 show MPEVR produced when kriging is applied to compressed data under different configurations of the parameters 
Table 1: ANOVA on RMSPE's for all the significant factors and interactions.

(a) K-adaptive vs. K-local

(b) K-adaptive vs. K-binned

\begin{tabular}{|c|c|c|c|c|c|c|c|c|c|}
\hline Factor & SS & $\overline{\mathrm{DF}}$ & $\mathrm{F}$ & p-value & Factor & SS & DF & $\mathrm{F}$ & $\mathrm{p}$-value \\
\hline $\mathrm{RD}$ & 0.13 & 1 & 18.18 & 0.00 & DR & 0.41 & 1 & 51.85 & 0.00 \\
\hline SNR & 12.72 & 4 & 434.31 & 0.00 & SNR & 8.05 & 4 & 255.23 & 0.00 \\
\hline$\theta$ & 542.08 & 3 & $24,668.68$ & 0.00 & $\theta$ & 581.74 & 3 & $24,587.35$ & 0.00 \\
\hline $\mathrm{DR} \times \mathrm{SNR}$ & 0.23 & 4 & 7.87 & 0.00 & $\mathrm{DR} \times \mathrm{SNR}$ & 0.08 & 4 & 2.42 & 0.05 \\
\hline $\mathrm{DR} \times \theta$ & 0.09 & 3 & 4.18 & 0.01 & $\mathrm{DR} \times \theta$ & 0.31 & 3 & 12.97 & 0.00 \\
\hline $\mathrm{SNR} \times \theta$ & 0.49 & 12 & 5.53 & 0.00 & $\mathrm{SNR} \times \theta$ & 0.94 & 12 & 9.95 & 0.00 \\
\hline Residual & 58.31 & 7960 & & & Residual & 62.78 & 7960 & & \\
\hline
\end{tabular}

$\theta, \sigma_{\epsilon}^{2}$ (equivalently $\mathrm{SNR}$ ), and $K$. The closer the ratios are to one, the more accurate are the prediction variances produced using compressed data.

Each of the nine panels in Figure 5 corresponds to a unique combination of $\theta$ and SNR. Within each panel, the first boxplot labeled K-full is always equal to 1. For K-adaptive, K-local, and K-binned, there are two boxplots each; one corresponds to compression with $K=200$ (coarse compression), and one to $K=600$ (fine compression). In all panels, the dashed horizontal line is for K-full, the benchmark. The K-adaptive MPEVR is closest to 1 in almost all cases. In panel $5 \mathrm{c}$, where both SNR and $\theta$ are small, K-local does well for larger $K$, but $\mathrm{K}$-adaptive is competitive. These conditions are closest to having a very weak spatial structure relative to the noise level and are in line with our results for RMSPE in Figure 4. Once again, K-adaptive is the most robust choice over the wide range of possible conditions given in the simulation.

\subsection{Analysis of variance}

In this section, we quantify the results that are inferred from graphs presented in Section 3.3 through a formal analysis of variance (ANOVA). The factors are data reduction (DR) methodology, spatial scale $(\theta)$, and SNR, and the response variable is RMSPE. Since the RMSPE's for the simulation studies were approximately symmetrically distributed, a multi-factor ANOVA is appropriate for this study. Table 1 shows the main factors and all significant two-way interactions. We further separated the analysis to compare K-adaptive to K-local in Table 1a and to K-binned in Table 1b. All the factors, including data-reduction method, spatial scale, and SNR have large F-statistics for both sets of comparisons. The significance of the interaction effects is slightly different for K-adaptive compared to K-local versus $\mathrm{K}$-adaptive compared to K-binned. Interaction $\mathrm{DR} \times \mathrm{SNR}$ is stronger for $\mathrm{K}$-adaptive versus K-local, confirming that noisy data have a greater impact on the performance of 
K-local. On the other hand, the interaction $\mathrm{DR} \times \theta$ for $\mathrm{K}$-adaptive versus K-binned indicates that K-binned will be impacted to a greater degree by spatial scale (i.e., length of spatial correlations). Overall, the main factors and interactions all confirm that K-adaptive provides significant improvements over the other data reduction strategies.

The principal results of the simulation experiments can be summarized as follows:

- SNR is the most important factor and spatial scale is the second most important factor determining the performance of kriging applied to compressed data.

- K-adaptive (ASDC) has the most stable and consistent performance across all scenarios; this is an advantage when working with real data, where the true properties of the spatial field are unknown. By comparison, K-local and K-binned exhibit opposite trends in performance: K-local performs worse for small SNR and large spatial scale parameters, and K-binned performs worse for large SNR and small spatial scale parameters.

- K-adaptive (ASDC) produces the most reliable prediction variances.

- K-adaptive (ASDC) performs well for very coarse levels of compression and can be effective for when drastic data reduction is needed.

\subsection{Computational costs}

In this section we compare the computational cost of K-full and that of ASDC. Computational complexity of kriging is $O\left(n^{3}\right)$, where $n$ is the number of observed locations. Since the compressed dataset is much smaller in size than the full data, the computational cost of kriging itself based on compressed data become negligible. Therefore, we focus here on computational costs of ASDC. We do not consider the costs of fitting the spatial dispersion function as they would be the same for both full kriging (fitting the variogram) and ASDC.

The costs of ASDC can be roughly separated into three components: the construction of the similarity matrix, the eigenvalue decomposition, and the $K$-means algorithm. The computational cost of a standard $K$-means algorithm (e.g., Hartigan and Wong (1979)) is considered linear in practice (Jain et al., 1999; Duda et al., 2012); for ASDC, it will depend linearly on the number of spatial locations in $\mathcal{D}_{e}, N-M$, and on the number of clusters, $K$, which is very small. The eigenvalue decomposition is $O\left((N-M)^{3}\right)$ in computational complexity, however sparse matrix techniques can be used to bring it down to be approximately between linear and quadratic, i.e., $O((N-M) K)$ (Song et al., 2008), with $K \ll n<N$. Finally, the construction of the similarity matrix is $O\left((N-M)^{2}\right)$, but a large, 
dense matrix $\mathbf{H}$ must be computed initially and will likely challenge memory limitations when $N$ is large. Thus, the computational bottleneck for ASDC is the construction and storage of the similarity matrix. This can be addressed by constructing $\mathbf{H}$ as a sparse matrix (Hastie et al., 2015), and options such as rank or dimension reduction can be employed (e.g., Katzfuss, 2016). A detailed analysis of computational complexity and memory usage in spectral clustering, including sparse matrix eigen-decomposition, can be found in Song et al. (2008).

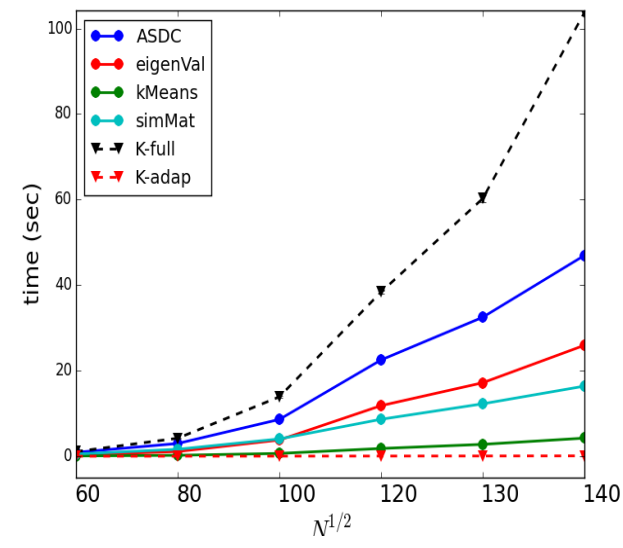

(a) Computation time (seconds) vs. size of the spatial domain.

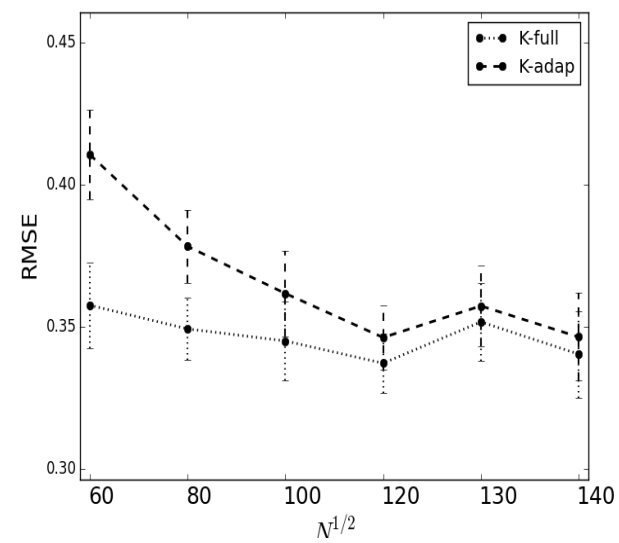

(c) RMSPE vs. size of spatial domain.

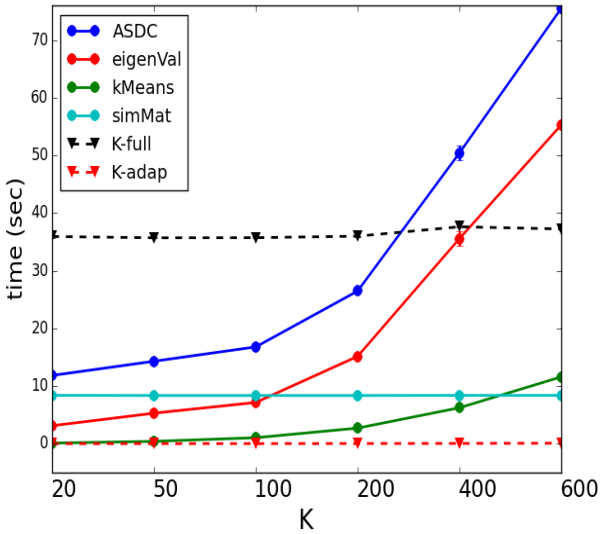

(b) Computation time (seconds) vs. number of clusters $K$ for a square domain of size $120 \times 120$.

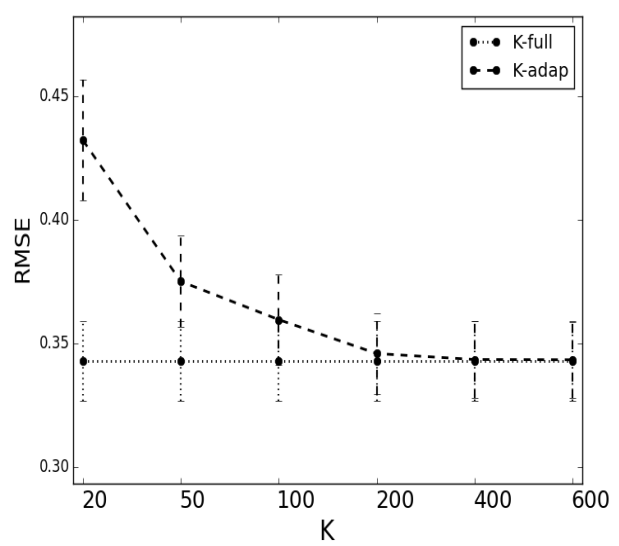

(d) RMSPE vs. the number of clusters $K$ for a square domain of size $120 \times 120$.

Figure 6: Computational costs and RMSPE of K-full vs. K-adaptive (ASDC).

Figure 6 shows our analysis of computation times for K-full and K-adaptive (ASDC) in our synthetic example. Figure 6a shows computation time in seconds as a function of 
increasing numbers of spatial locations, $N=\left\{60^{2}, 80^{2}, 100^{2}, 120^{2}, 130^{2}, 140^{2}\right\}$, for K-full and for K-adaptive with $99 \%$ target compression $(K=200)$. The overall computation time for ASDC (blue) is further separated into the construction of the similarity matrix (cyan), the eigenvalue decomposition (red), and the $K$-means algorithm (green). The times are averages over $B=100$ randomly generated spatial fields, for each value of $N$, as in Section 3.1. The parameters of the exponential model are fixed at $\theta=0.5$ and $\mathrm{SNR}=100$, with no missing data. Additional experiments (not described here) show that other choices of these parameters do not greatly affect computation time. The computational costs of kriging based on ASDC-compressed data (red dotted line) are negligible. Overall, Figure 6a shows that ASDC (solid blue line) scales better than K-full (dashed black line) as the data size increases. Figure 6c shows the corresponding RMSPE averages and 95\% confidence intervals for K-full and K-adaptive. K-adaptive RMSPE's converge to those for K-full as $N$ increases. The sizes of the compressed datasets also increase slightly, but the compression ratio remains constant, i.e., 99\%. This indicates that as the size of the data increases, ASDC could efficiently preserve the information contained in the full dataset at a proportionally decreasing computational cost.

Figure 6b shows how ASDC computation time for a dataset of size $N=120^{2}$ varies with decreasing compression level, i.e., as the number of clusters, $K$, increases. ASDC overall computation time is decomposed as in Figure 6a. The overall computation time (blue) increases approximately cubically, and is driven primarily by the eigenvalue decomposition (red). ASDC's computation time reaches K-full's computation time at about $K=300$, which is about $98 \%$ compression. Figure $6 \mathrm{~d}$ shows that the accuracy of the prediction based on ASDC, as measured by RMSPE, reaches that of K-full at about $K=200$ or slightly under $99 \%$ compression. This shows that ASDC can reduce computational costs of kriging on large datasets with relatively small loss of information content and predictive performance. We provide an additional discussion of computational challenges in Section 5.

\section{Sea-surface temperature (SST) data}

In this section we demonstrate the use of ASDC on a modest set of data from the Advanced Microwave Scanning Radiometer 2 instrument (AMSR-2; Wentz et al. (2014)) in order to show how ASDC performs when we do not know the true spatial model. These data are representative of spatial structure and patterns of missingness that are characteristic of remote sensing data. We focus on a $50^{\circ} \times 50^{\circ}$ region in the north Atlantic ocean just off the east coast of the US and Canada as shown in Figure 7 . We call this area the Gulf 
Stream region, and it is important because of large fish populations in the area. The Gulf Stream region includes some coastlines; these areas are particularly important because this is where upwelling (the movement of cold, nutrient-rich water towards the surface) occurs. Upwelling information is an important predictor of fishery productivity and ocean-circulation (Vazquez-Cuervo et al., 2013).

We subdivide this region into into nine equal-size, non-overlapping square subregions, delineated by the black lines in Figure 7a. For each subregion, we make three sets of kriging predictions, one each using ASDC-compressed data (K-adaptive), data binned to a coarse resolution (K-binned), and data in a local neighborhood (K-local). The data is compressed only in the exterior and is retained as is in the prediction region. We then compare these predictions to the full-data prediction (K-full), depicted in Figure $7 \mathrm{~b}$, as we did in the simulation study presented in Section 3.3.

The reason for breaking the domain into nine separate subdomains is twofold. First, the spatial distributions of observations within these subdomains are different, and collectively they are representative of the kinds of patterns of missingness one often encounters in remote sensing. For example, subdomains 3 and 8 have relatively good data coverage, with missing data concentrated in relatively small contiguous areas because the missingness is caused by

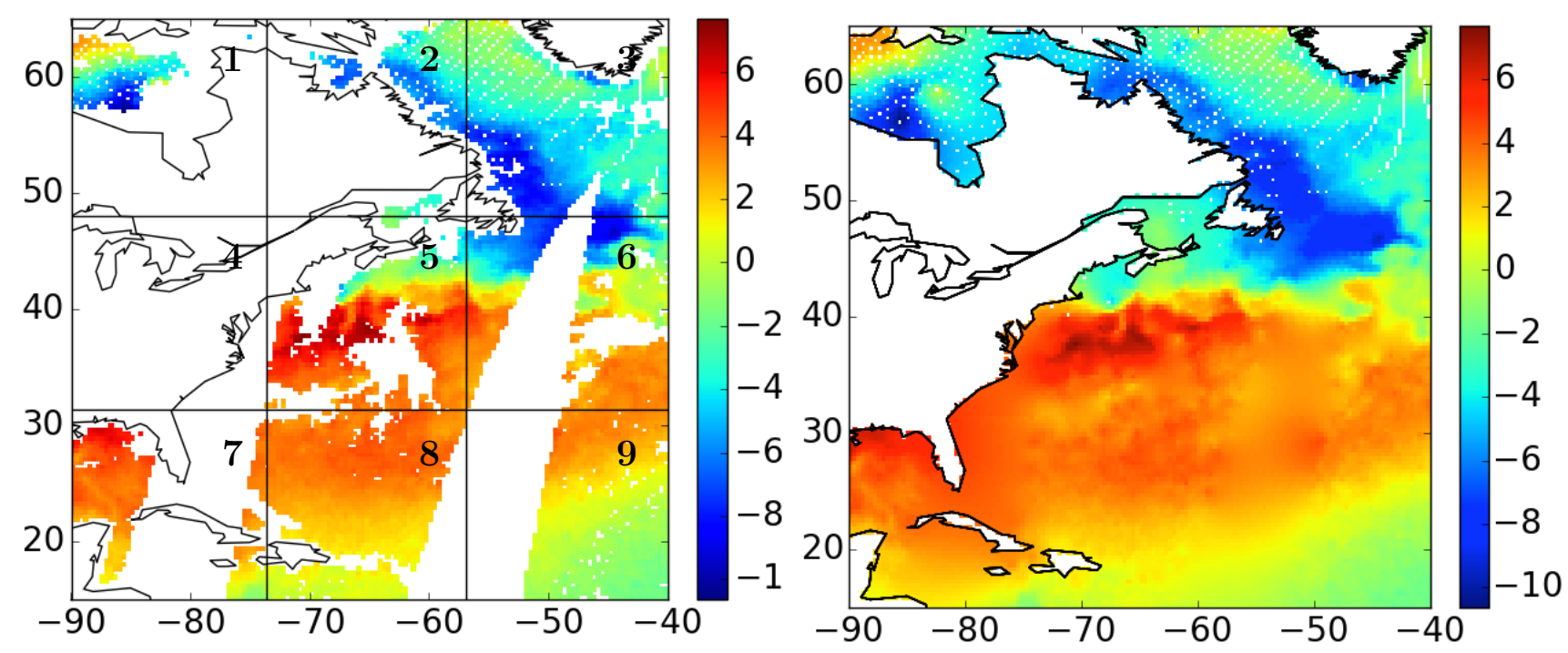

(a) Detrended AMSR-2 SST, daytime on July $13,2015$.

(b) Predictions based on K-full.

Figure 7: Detrended SST and corresponding kriging predictions based on the full dataset. The color scale is in ${ }^{\circ} \mathrm{K}$. 
areas between satellite overpasses. Subdomains 6 and 9 are missing observations over about half their domains; in subregion 6 the missing area is approximately in the center, while in subdomain 9 it is all to one side, also due to the satellite orbit. Subdomain 7 is similar to subdomain 6 , but with a larger proportion of missingness; subdomain 5 has missing data due to both coastline and precipitation, through which AMSR-2 can not see. Subdomains 1 and 2 have quite a bit more missing data, and also appear to be missing data for reasons similar to that of subdomain 5. Finally, subdomain 4 is almost entirely missing, but does have a few observed data points in the ocean. To assess the impact of these different patterns, we report RMSPE and MPEVR for each region separately in Table 2 below.

The second reason for breaking the domain into subdomains is to demonstrate what happens when data are processed in pieces. This may be required when data volumes are truly massive in order to exploit parallel processing. This "chunking" can produce edge effects when the nine kriged subdomains are recombined to create a single kriged data set. To quantify this effect, our RMSPE and MPEVR figures of merit are also reported for the entire domain as a whole in Table 2.

In this analysis there are 11,800 AMSR-2 observations in the Gulf Stream region during the daytime on July 19, 2015. We detrended these data using a quadratic function of latitude, since this approximately captures the way SST decays moving from the Equator to the poles. AMSR-2 footprints are $25 \mathrm{~km}^{2}$ ellipses and we matched their centers to the centers of hexagonal grid cells at $30 \mathrm{~km}^{2}$ resolution (Sahr et al. (2003); discrete global grid resolution 8) superimposed on the domain. The benefits of using the hexagonal grid over a rectangular grid are well-known (Birch et al., 2007). The centers of these hexagonal cells are the prediction locations for kriging, and the subdomains contain between about 450 and 4,100 ocean prediction locations, each. Finally, $K$ was set to 100 in all cases.

We estimated the parameters of an exponential covariance model from all data in the Gulf Stream region, and performed kriging on the full dataset (K-full) and on data compressed using ASDC (K-adaptive), subsetting (K-local), and binning (K-binned) as we did in the simulation study. The same covariance model was used in all four cases to avoid confounding data compression performance assessments with the quality of the covariance function estimate. The covariance function parameter estimates are $\hat{\theta}=23.6$ and $\hat{\sigma}_{Y}^{2}=43.7$ $\left({ }^{\circ} K\right)^{2}$ and are obtained by fitting the exponential function with curve_fit of the scipy Python library.

Table 2 shows RMSPE and MPEVR, defined in (16) and (17), respectively, with $y_{i}$ now being the kriging prediction, based on the full dataset, for each of the nine subdomains. 
Table 2: RMSPE and mean prediction error variance ratio (MPEVR)

\begin{tabular}{c|lll|lll}
\hline \multirow{2}{*}{ Subregion } & \multicolumn{3}{|c|}{ RMSPE } & \multicolumn{3}{c}{ MPEVR } \\
& K-adaptive & K-local & K-binned & K-adaptive & K-local & K-binned \\
\hline 1 & $0.036^{*}$ & 0.501 & 0.079 & 0.997 & 1.045 & $1.000^{*}$ \\
2 & $0.162^{*}$ & 0.180 & 0.180 & $0.994^{*}$ & 1.014 & 1.018 \\
3 & $0.042^{*}$ & 0.084 & 0.078 & $1.002^{*}$ & $1.002^{*}$ & 1.005 \\
\cline { 2 - 7 } 4 & $0.389^{*}$ & 0.983 & 0.606 & 1.125 & $1.101^{*}$ & 1.417 \\
5 & 0.037 & 0.069 & $0.029^{*}$ & $1.002^{*}$ & 1.004 & 1.010 \\
6 & $0.048^{*}$ & 0.094 & 0.070 & $1.003^{*}$ & 1.019 & 1.006 \\
\cline { 2 - 7 } 7 & $0.055^{*}$ & 0.157 & 0.085 & $1.000^{*}$ & 1.056 & 1.006 \\
8 & $0.044^{*}$ & 0.081 & 0.092 & $1.003^{*}$ & 1.017 & 1.004 \\
9 & $0.043^{*}$ & 0.118 & 0.142 & 0.994 & 1.052 & $1.004^{*}$ \\
\hline all & $0.078^{*}$ & 0.208 & 0.130 & $1.002^{*}$ & 1.029 & 1.014 \\
\hline
\end{tabular}

The top nine rows of Table 2 summarize the performance of the three data compression methods for each of the nine prediction subregions, with starred values highlighting the best performance. The last row of the table shows performance when the kriged estimates for the nine subregions are recombined into one field. K-adaptive has the lowest RMSPE for all subdomains other than subdomain 5, where K-binned is slightly better. Recall that subdomain 5 is the only subdomain in which a significant portion of the missing data are surrounded by observed data. The MPVER for K-adaptive is best in six of the nine subdomains, including subdomain 5. Where K-adaptive's performance metrics are slightly inferior to those of K-local or K-binned, it is only by a very small margin, and most likely due to the natural variation in the data. Our conclusion is that when the true pattern of missingness is not of the most favorable form, i.e., spherical, central in the domain, and not exceeding more than about half the locations, K-adaptive tends to be superior to the traditional data reduction methods. The last row of the table combines the predictions and their uncertainties for all nine regions, and compares them to the full-kriging result. Here, K-adaptive is best in both RMSPE and MPVER, which is not surprising given the results on the individual subdomains.

Finally, Figure 8 shows the spatial predictions and their standard errors in the Gulf Stream region for $K=100$ for K-adaptive in the first column and for K-local and K-binned in the second and third columns, respectively. Differences in the predicted values across the three compression methods are small, especially at locations with corresponding observed values. Differences are evident, however, in the areas where large blocks of data are missing between satellite swaths. These differences are due to edge effects that arise when prediction is performed on several non-overlapping subregions. When K-full is used, the edge effects 

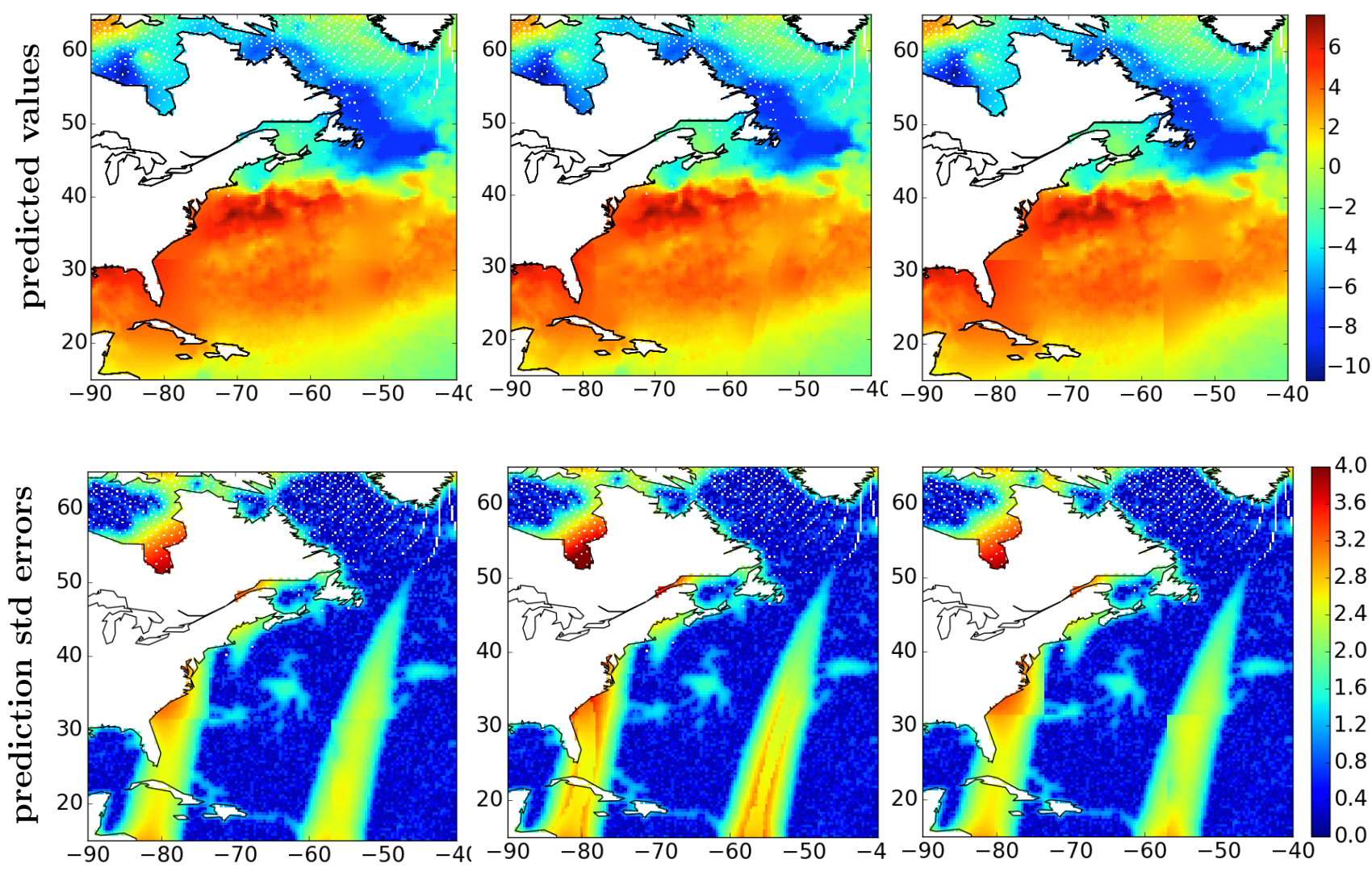

(a) K-adaptive

(b) K-local

(c) K-binned

Figure 8: Combined predicted SST values and their standard errors for the Gulf Stream for K-adaptive, K-local, and K-binned for a reduced dataset of size $K=100$. (The regular pattern of white (missing) pixels between Labrador and Greenland are an artifact of projecting AMSR-2 data from the rectangular to hexagonal grid.

are mostly eliminated as seen in Figure $7 \mathrm{~b}$. Thus, in addition to its generally superior ability to preserve information, ASDC is also reduces the impact of edge effects when data are processed in chunks. The prediction standard errors obtained with K-adaptive are the closest to those of K-full as shown in Table 2. Figure 8 shows that the K-adaptive and $\mathrm{K}$-binned yield lower prediction errors in the areas between swaths and in coastal regions where K-local prediction variances can be very large.

\section{Summary, conclusions, and future work}

In this paper, we introduced ASDC, a spatial data compression method that explicitly preserves the spatial covariance structure, and enables substantial size reductions of datasets to be used for spatial inference. We showed how ASDC uses the machinery of spectral 
clustering to capture two kinds of spatial correlations. The first are those between locations outside the area in which spatial predictions are to be made, and the second are those between locations inside and outside. This strategy leads to efficient compression of the data outside while preserving information that is relevant to estimation inside the prediction area.

We studied the performance of ASDC by comparing it to two traditional methods for reducing the size of spatial data sets, subsetting and binning, in a simulation study. We generated ensembles of 100 randomly generated synthetic spatial fields from an exponential covariance model with known parameters, added measurement noise, compressed the data outside a central region of interest using ASDC and the two competitors, and then performed kriging to predict the data values in the region of interest. Performance is quantified through the distributions of two figures of merit over the 100-member ensemble: RMSPE, a measure of accuracy of the kriging predictions relative to the true simulated spatial field; and MPEVR, a measure of the accuracy of the kriging prediction variance, relative to that which is obtained when the original data outside are used. This experiment was conducted for various scenarios in which we used different spatial scale parameters in the exponential model used to generate the synthetic field, and different levels of measurement noise.

An important practical conclusion from this study is that ASDC is more robust to changes in spatial correlation in the underlying field, and to measurement error, than are its competitors. This robustness exists for both accuracy of prediction measured by RMSPE and, to a slightly lesser degree, to the accuracy of the prediction error measured by MPEVR. Robustness is an important advantage since we do not know the true spatial scale parameter when we work with real data. We caution, however, that this conclusion is drawn from experiments that assume an exponential covariance function. A logical next step in the simulation study is to use a different covariance model to generate synthetic spatial fields.

We used an analysis of variance to quantify the effects of the different factors in the experiment in head-to-head comparisons of the ASDC against subsetting and binning, and found that all factors (spatial scale parameter, measurement error variance, data compression method and all their interactions) are statistically significant. In fact, the most important factors are the spatial scale parameter used to generate the field and the measurement noise.

To explore how computational costs scale with dataset size and degree of compression, we looked at computation times for the compound task of ASDC data compression followed by kriging. Computation time for ASDC followed by kriging is dominated by the construction of the similarity matrix and eigenvalue decomposition required by spectral clustering, but 
even the total time does not go up as fast as that of kriging the full dataset. As a function of the number of spatial clusters, computation time does not rise substantially until the number of clusters reaches about 200 in our study. At the same time the RMSPE (versus truth) of the kriging predictor that uses ASDC to compress the data outside the region of interest, converges to that of kriging on the full dataset at the same number of clusters, namely $K=200$. These results suggest that a "sweet spot" exists for ASDC compression. In future work we will explore how that sweet spot depends on the structure of the underlying spatial field and on measurement error.

Finally, we applied ASDC and competitors to sea-surface temperature data from NASA's AMSR-2 instrument by breaking these data into nine separate subregions. The subregions have different patterns of missing data, and part of the objective was to assess how these patterns might affect performance. RMSPE and MPEVR reported in Table 2 show that ASDC is also more robust to missing data than are subsetting and binning; this represents another important advantage of ASDC, since patterns of missingness in remote sensing data can depend on changing observing conditions. When the kriging predictions for the nine separate subregions were recombined, the resulting maps showed fewer edge effects when ASDC was used to pre-compress the data than were present when the alternative procedures were used. If this result is not unique to the range of experimental conditions used in this study, then it should be possible to break large datasets into non-overlapping regions and apply $\mathrm{ASDC}$ on those regions in parallel.

We plan additional studies to expand the range of experimental conditions used in our simulations, including other spatial covariance functions for generating synthetic fields, and modified spatial dispersion functions to incorporate geometric anisotropy (Zimmerman, 1993; Sherman, 2011) in cluster formation. A number of other optimizations to our current algorithm, including exploitation of sparsity, low-rank approximations, and parallel processing, are on the horizon and will be necessary in order to perform ASDC on truly massive datasets in an operational data processing environment.

\section{Acknowledgements}

This work was performed at the Jet Propulsion Laboratory, California Institute of Technology under contract with the National Aeronautics and Space Administration. (C) Copyright 2016, California Institute of Technology. All rights reserved. The authors would like to thank Drs. Michael Turmon, Jeffrey Jewell, Jorge Vazquez, Jonathan Hobbs, and Vineet Yadav for their helpful comments and discussion. Cressie's research was partially supported by NASA 
grant NNHII-ZDA001N-OCO2, and by a 2015-2017 Australian Research Council Discovery Project, DP150104576.

Ambroise, C., Dang, M., Govaert, G., 1997. Clustering of spatial data by the EM algorithm. geoENV I Geostatistics for Environmental Applications, 493-504.

Arthur, D., Vassilvitskii, S., 2007. K-means++: The advantages of careful seeding. In: Proceedings of the Eighteenth Annual ACM-SIAM Symposium on Discrete Algorithms. Society for Industrial and Applied Mathematics, pp. 1027-1035.

Banerjee, S., Gelfand, A., Finley, A., Sang, H., 2008. Gaussian predictive process models for large spatial data sets. Journal of the Royal Statistical Society: Series B (Statistical Methodology) 70 (4), 825-848.

Besag, J., Kooperberg, C., 1995. On conditional and intrinsic autoregressions. Biometrika 82, 733-746.

Birch, C., Oom, S. P., Beecham, J. A., 2007. Rectangular and hexagonal grids used for observation, experiment and simulation in ecology. Ecological Modelling 206 (3), 347-359.

Bornn, L., Shaddick, G., Zidek, J., 2012. Modeling non-stationary processes through dimension expansion. Journal of the American Statistical Association 107 (497), 281-289.

Chen, W., Song, Y., Bai, H., Lin, C., Chang, E., 2011. Parallel spectral clustering in distributed systems. Pattern Analysis and Machine Intelligence, IEEE Transactions on, 33 (3), 568-586.

Chung, F., 1997. Spectral graph theory (Vol. 92). American Mathematical Soc.

Craddock, R., James, G., Holtzheimer, P., Hu, X., Mayberg, H., 2012. A whole brain fMRI atlas generated via spatially constrained spectral clustering. Human Brain Mapping 33 (8), 1914-1928.

Cressie, N., 1993. Statistics for Spatial Data. New York, NY, Wiley.

Cressie, N., Johannesson, G., 2008. Fixed rank kriging for very large spatial datasets. Journal of the Royal Statistical Society: Series B (Statistical Methodology) 70 (1), 209-226.

Datta, A., Banerjee, S., Finley, A., Gelfand, A., 2015. Hierarchical nearest-neighbor Gaussian process models for large geostatistical datasets. Journal of the American Statistical Association (just-accepted) 00 (00), 00-00.

Duda, R., Hart, P., Stork, D., 2012. Pattern classification. John Wiley \& Sons.

Eidsvik, J., Shaby, B., Reich, B., Wheeler, M., Niemi, J., 2014. Estimation and prediction in spatial models with block composite likelihoods. Journal of Computational and Graphical Statistics 23 (2), 295-315.

Finley, A., Banerjee, S., McRoberts, R., 2009. Hierarchical spatial models for predicting tree species assemblages across large domains. Annals of Applied Atatistics 3 (3), 1052.

Furrer, R., Genton, M., Nychka, D., 2006. Covariance tapering for interpolation of large spatial datasets. Journal of Computational and Graphical Statistics 15 (3), 502-523.

Gramacy, R., Apley, D., 2015. Local Gaussian process approximation for large computer experiments. Journal of Computational and Graphical Statistics 24 (2), 561-578.

Haas, T., 1990. Kriging and automated variogram modeling within a moving window. Atmospheric Environment. Part A. General Topics 24 (7), 1759-1769.

Hammerling, D., Michalak, A., Kawa, S., 2012. Mapping of CO2 at high spatiotemporal resolution using satellite observations: Global distributions from OCO-2. Journal of Geophysical Research: Atmospheres 117 (D6).

Hartigan, J., Wong, M., 1979. Algorithm AS 136: A K-means clustering algorithm. Journal of the Royal Statistical Society, Series C (Applied Statistics) 28 (1), 100-108. 
Hastie, T., Tibshirani, R., Wainwright, M., 2015. Statistical learning with sparsity: the lasso and generalizations. CRC Press.

Hu, T., Sung, S., 2006. A hybrid EM approach to spatial clustering. Computational Statistics and Data Analysis 50 (5), 1188-1205.

Jain, A., Murty, M., Flynn, P., 1999. Data clustering: a review. ACM computing surveys (CSUR) 31 (3), 264-323.

Katzfuss, M., 2016. A multi-resolution approximation for massive spatial datasets. Journal of the American Statistical Association (forthcoming).

Kaufman, C., Schervish, M., Nychka, D., 2008. Covariance tapering for likelihood-based estimation in large spatial data sets. Journal of the American Statistical Association 103 (484), 1545-1555.

Lindgren, F., Rue, H., Lindström, J., 2011. An explicit link between gaussian fields and gaussian markov random fields: the stochastic partial differential equation approach. Journal of the Royal Statistical Society: Series B (Statistical Methodology) 73 (4), 423-498.

Ng, A., Jordan, M., Weiss, Y., 2002. On spectral clustering: analysis and an algorithm. In: T. Dietterich, S. Becker, and Z. Ghahramani (Eds.) Advances in Neural Information Processing Systems. Vol. 14. MIT Press, pp. 849-856.

Nguyen, H., Cressie, N., Braverman, A., 2012. Spatial statistical data fusion for remote sensing applications. Journal of the American Statistical Association 107 (499), 1004-1018.

Nychka, D., Bandyopadhyay, S., Hammerling, D., Lindgren, F., Sain, S., 2015. A multi-resolution gaussian process model for the analysis of large spatial datasets. Journal of Computational and Graphical Statistics 24 (2), 579-599.

Papp, D., Alizadeh, F., 2014. Shape-constrained estimation using nonnegative splines. Journal of Computational and Graphical Statistics 23 (1), 211-231.

Rue, H., Held, L., 2005. Gaussian Markov random fields: theory and applications. CRC Press.

Sahr, K., White, D., Kimerling, A., 2003. Geodesic discrete global grid systems. Cartography and Geographic Information Science 30 (2), 121-134.

Sampson, P., Guttorp, P., 1992. Nonparametric estimation of non-stationary spatial covariance structure. Journal of the American Statistical Association 87 (417), 108-119.

Sang, H., Huang, J., 2012. A full scale approximation of covariance functions for large spatial data sets. Journal of the Royal Statistical Society, Series B (Statistical Methodology) 74 (1), 111-132.

Sherman, M., 2011. Spatial statistics and spatio-temporal data: covariance functions and directional properties. John Wiley \& Sons.

Shi, J., Malik, J., 2000. Normalized cuts and image segmentation. EEE Transactions on Pattern Analysis and Machine Intelligence 22 (8), 888-905.

Song, Y., Chen, W., Bai, H., Lin, C., Chang, E., 2008. Parallel spectral clustering. In: Joint European Conference on Machine Learning and Knowledge Discovery in Databases. Springer Berlin Heidelberg, pp. 374-389.

Tobler, W., 1970. A computer movie simulating urban growth in the detroit region. Economic Geography 46 (2), 234-240.

Vazquez-Cuervo, J., Dewitte, B., Chin, T., Armstrong, E., Purca, S., Alburqueque, E., 2013. An analysis of sst gradients off the peruvian coast: The impact of going to higher resolution. Remote Sensing of 
Environment 131, 76-84.

Von Luxburg, U., 2007. A tutorial on spectral clustering. Statistics and Computing 17 (4), 395-416.

Wentz, F., Meissner, T., Gentemann, C., Hilburn, K., J., S., 2014. Remote Sensing Systems GHRSST Level 2P Global Subskin Sea Surface Temperature from the Advanced Microwave Scanning Radiometer 2 on the GCOM-W satellite. Ver. 7.2. Remote Sensing Systems, PO.DAAC, CA, USA.

Wever, U., 1988. Non-negative exponential splines. Computer-aided Design 20 (1), 11-16.

Zare, H., Shooshtari, P., Gupta, A., Brinkman, R., 2010. Data reduction for spectral clustering to analyze high throughput flow cytometry data. BMC Bioinformatics 11 (1), 403.

Zelnik-Manor, L., Perona, P., 2004. Self-tuning spectral clustering. In: Advances in Neural Information Processing Systems. pp. 1601-1608.

Zimmerman, D., 1993. Another look at anisotropy in geostatistics. Mathematical Geology 25 (4), 453-470. 